\title{
Theory of valuations on manifolds, IV. New properties of the multiplicative structure.
}

\author{
Semyon Alesker * \\ Department of Mathematics, Tel Aviv University, Ramat Aviv \\ 69978 Tel Aviv, Israel \\ e-mail: semyon@post.tau.ac.il
}

\begin{abstract}
This is the fourth part in the series of articles [4], [5], 6] (see also [3]) where the theory of valuations on manifolds is developed. In this part it is shown that the filtration on valuations is compatible with the product. Then it is proved that the Euler-Verdier involution on smooth valuations is an automorphism of the algebra of valuations. Then an integration functional on valuations with compact support is introduced, and a property of selfduality of valuations is proved. Next a space of generalized valuations is defined, and some basic properties of it are proved. Finally a canonical imbedding of the space of constructible functions on a real analytic manifold into the space of generalized valuations is constructed, and various structures on valuations are compared with known structures on constructible functions.
\end{abstract}

\section{Contents}

\begin{tabular}{lll}
\hline 0 & Introduction. & 2
\end{tabular}

1 Background.

1.1 Notation. . . . . . . . . . . . . . . . . . . . 5



1.3 Characteristic and normal cvcles. . . . . . . . . . . . . . . . . 7

1.4 Some valuation theorv . . . . . . . . . . . . . . . . . . . . . . . . . . . . . . . . . . . .

1.5 Filtration on valuations. . . . . . . . . . . . . . . . . . . 12

2 A technical lemma. $\quad 14$

3 Compatibility of the filtration with the product. 16

4 The automorphism property of the Euler-Verdier involution. 17

*Partially supported by ISF grant 1369/04. 
$\begin{array}{lll}5 & \text { The integration functional on valuations. } & 19\end{array}$

5.1 Valuations with compact support. . . . . . . . . . . . . . . . . . 19

5.2 The integration functional. . . . . . . . . . . . . . . . . . . . 20

$6 \quad$ The selfduality property of valuations. 21

6.1 The selfdualitv propertv . . . . . . . . . . . . . . . . . 21

6.2 Partition of unity in valuations . . . . . . . . . . . . . . . . . 22

$\begin{array}{lll}7 & \text { Generalized valuations. } & 23\end{array}$

7.1 The space of generalized valuations. . . . . . . . . . . . . . . . 23

7.2 The sheaf propertv of generalized valuations. . . . . . . . . . . . . . . 24

7.3 Filtration on generalized valuations. . . . . . . . . . . . . . . 25

7.4 The Euler-Verdier involution on generalized valuations. . . . . . . . . . . . 32

8 Valuations on real analvtic manifolds. 33

8.1 Imbedding of constructible functions to generalized valuations. . . . . . . . . 33

8.2 Comparison of filtrations. . . . . . . . . . . . . . . 35

8.3 The integration functional vs. the integration with respect to the Euler characteristic. 37

8.4 The Euler-Verdier involution vs. the Verdier duality . . . . . . . . . . . 38

\section{Introduction.}

In convexity there are many geometrically interesting and well known examples of valuations on convex sets: Lebesgue measure, the Euler characteristic, the surface area, mixed volumes, the affine surface area. For a description of older classical developments on this subject we refer to the surveys 21], [20]. For the general background on convexity we refer to the book [23].

Approximately during the last decade there was a significant progress in this classical subject which has led to new classification results of various classes of valuations, to discovery of new structures on them. This progress has shed a new light on the notion of valuation which allowed to generalize it to more general setting of valuations on manifolds and on not necessarily convex sets (which do not make sense on a general manifold). On the other hand author's feeling is that the notion of valuation equips smooth manifolds with a new general structure. The development of the theory of valuations on manifolds was started in three previous parts of the series of articles: [4], [5] by the author and [6] by J. Fu and the author. This article in the forth part in this series.

In [5] the notion of smooth valuation on a smooth manifold was introduced. Roughly speaking a smooth valuation can be thought as a finitely additive $\mathbb{C}$-valued measure on a class of nice subsets; this measure is requested to satisfy some additional assumptions of continuity (or rather smoothness) in some sense. The basic examples of smooth valuations on a general manifold $X$ are smooth measures on $X$ and the Euler characteristic. Moreover the well known intrinsic volumes of convex sets can be generalized to provide examples of smooth valuations on an arbitrary Riemannian manifold; these valuations are known as Lipschitz-Killing curvatures. 
Let $X$ be a smooth manifold of dimension $n$. The space of smooth valuations on $X$ is denoted by $V^{\infty}(X)$. It has a canonical linear topology with respect to which it becomes a Fréchet space.

The space $V^{\infty}(X)$ carries a canonical multiplicative structure. This structure seems to be of particular interest and importance. When $X$ is an affine space it was defined in [4] (in even more specific situation of valuations polynomial with respect to translations it was defined in [3]). For a general manifold $X$ the multiplicative structure was defined in [6]. The construction in [6] uses the affine case [4] and additional tools from the geometric measure theory.

It was shown in [6] that the product $V^{\infty}(X) \times V^{\infty}(X) \rightarrow V^{\infty}(X)$ is a continuous map, and $V^{\infty}(X)$ becomes a commutative associative algebra with the unit (which is the Euler characteristic). The goal of this article is to study further properties of the multiplicative structure and apply one of them (which we call the Selfduality property) to introduce a new class of generalized valuations.

In [5] a filtration of $V^{\infty}(X)$

$$
V^{\infty}(X)=W_{0} \supset W_{1} \supset \cdots \supset W_{n}
$$

by closed subspaces was introduced. The first main result of this article (Theorem 3.1.1) says that this filtration is compatible with the product, namely $W_{i} \cdot W_{j} \subset W_{i+j}$ (where $W_{k}=0$ for $k>n)$.

In [5] the author has introduced a continuous involution $\sigma: V^{\infty}(X) \rightarrow V^{\infty}(X)$ called the Euler-Verdier involution. The second main result of this article says that $\sigma$ is an algebra automorphism (Theorem 4.1.4).

Let us denote by $V_{c}^{\infty}(X)$ the space of compactly supported smooth valuations. Next we introduce in this article the integration functional $\int: V_{c}^{\infty}(X) \rightarrow \mathbb{C}$. Slightly oversimplifying, it is defined by $[\phi \mapsto \phi(X)]$. The third main result is as follows.

0.1.1 Theorem. Consider the bilinear form

$$
V^{\infty}(X) \times V_{c}^{\infty}(X) \rightarrow \mathbb{C}
$$

given by $(\phi, \psi) \mapsto \int \phi \cdot \psi$.

This bilinear form is a perfect pairing. More precisely the induced map

$$
V^{\infty}(X) \rightarrow\left(V_{c}^{\infty}(X)\right)^{*}
$$

is injective and has a dense image with respect to the weak topology on $\left(V_{c}^{\infty}(X)\right)^{*}$.

This is Theorem 6.1.1 in the paper. Its proof uses the Irreducibility Theorem from [2] in full generality. Roughly Theorem 0.1 .1 can be interpreted as a selfduality property of the space of valuations (at least when the manifold $X$ is compact).

Let us denote $V^{-\infty}(X):=\left(V_{c}^{\infty}(X)\right)^{*}$. We call $V^{-\infty}(X)$ the space of generalized valuations. We show (Proposition [7.1.3) that $V^{-\infty}(X)$ has a canonical structure of $V^{\infty}(X)$ module.

In [5] it was shown that the assignment to any open subset $U \subset X$

$$
U \mapsto V^{\infty}(U)
$$


with the natural restriction maps is a sheaf denoted by $\mathcal{V}_{X}^{\infty}$. Here we show that

$$
U \mapsto V^{-\infty}(U)
$$

with the natural restriction maps is also a sheaf which we denote by $\mathcal{V}_{X}^{-\infty}$. Moreover $\mathcal{V}_{X}^{-\infty}$ is a sheaf of $\mathcal{V}_{X}^{\infty}$-modules (Proposition [7.2.4).

Remind that by [5] the last term $W_{n}$ of the filtration (0.1.1) coincides with the space $C^{\infty}\left(X,\left|\omega_{X}\right|\right)$ of smooth densities on $X$ (where $\left|\omega_{X}\right|$ denotes the line bundle of densities on $X)$, and $V^{\infty}(X) / W_{1}$ is canonically isomorphic to the space of smooth functions $C^{\infty}(X)$. In Subsection $\left[7.3\right.$ of this article we extend the filtration $\left\{W_{\bullet}\right\}$ to generalized valuations by taking the closure of $W_{i}$ in the weak topology on $V^{-\infty}(X)$ :

$$
V^{-\infty}(X)=W_{0}\left(V^{-\infty}(X)\right) \supset W_{1}\left(V^{-\infty}(X)\right) \supset \cdots \supset W_{n}\left(V^{-\infty}(X)\right) .
$$

We show that $W_{n}\left(V^{-\infty}(X)\right)$ is equal to the space $C^{-\infty}\left(X,\left|\omega_{X}\right|\right)$ of generalized densities on $X$ (Proposition [7.3.5). It is also shown that $V^{-\infty}(X) / W_{1}\left(V^{-\infty}(X)\right)$ is canonically isomorphic to the space $C^{-\infty}(X)$ of generalized valuations on $X$ (Proposition [7.3.6).

The Euler-Verdier involution is extended by continuity in the weak topology to the space of generalized valuations (Subsection 7.4). Also the integration functional extends (uniquely) by continuity in an appropriate topology to generalized valuations with compact support (Subsection 7.4).

In Section 8 we consider valuations on a real analytic manifold $X$. On such a manifold one has the algebra of constructible functions $\mathcal{F}(X)$ which is a quite well known object (see [18], Ch. 9). We construct a canonical imbedding of the space $\mathcal{F}(X)$ to the space of generalized valuations $V^{-\infty}(X)$ as a dense subspace. It turns out to be possible to interpret some properties of valuations in more familiar terms of constructible functions. Thus we show that the canonical filtration on $V^{-\infty}(X)$ induces on $\mathcal{F}(X)$ the filtration by codimension of support (Proposition 8.2.2). The restriction of the integration functional to the space of compactly supported constructible functions coincides with the well known functional of integration with respect to the Euler characteristic (Proposition 8.3.1). The restriction of the Euler-Verdier involution on $V^{-\infty}(X)$ to $\mathcal{F}(X)$ coincides (up to a sign) with the well known Verdier duality operator (Proposition 8.4.1).

Acknowledgements. I express my gratitude J. Bernstein for numerous stimulating discussions. I thank V.D. Milman for his attention to this work. I thank A. Bernig for sharing with me the recent preprint [7, J. Fu for very helpful explanations on the geometric measure theory, P.D. Milman for useful correspondences regarding subanalytic sets, and P. Schapira for useful discussions on constructible sheaves and functions.

\section{Background.}

In this section we fix some notation and remind various known facts. This section does not contain new results.

In Subsection 1.1 we fix some notation and remind the notions of normal and characteristic cycles of convex sets. In Subsection 1.2 we review basic facts on subanalytic sets. Subsection 1.3 collects facts on normal and characteristic cycles. In Subsection 1.4 we review some notions on valuations on manifolds following mostly [4, [5], [6]. Subsection [1.5] is also on valuations, and it reviews the canonical filtration on valuations following $[5]$. 


\subsection{Notation.}

Let $V$ be a finite dimensional real vector space.

- Let $\mathcal{K}(V)$ denote the family of convex compact subsets of $V$.

- Let $\mathbb{R}_{\geq 0}$ (resp. $\mathbb{R}_{>0}$ ) denote the set of non-negative (resp. positive) real numbers.

- For a manifold $X$ let us denote by $\left|\omega_{X}\right|$ the line bundle of densities over $X$.

- For a smooth manifold $X$ let $\mathcal{P}(X)$ denote the family of all simple subpolyhedra of $X$. (Namely $P \in \mathcal{P}(X)$ iff $P$ is a compact subset of $X$ locally diffeomorphic to $\mathbb{R}^{k} \times \mathbb{R}_{\geq 0}^{n-k}$ for some $0 \leq k \leq n$. For a precise definition see [5], Subsection 2.1.)

- We denote by $\mathbb{P}_{+}(V)$ the oriented projectivization of $V$. Namely $\mathbb{P}_{+}(V)$ is the manifold of oriented lines in $V$ passing through the origin.

- For a vector bundle $E$ over a manifold $X$ let us denote by $\mathbb{P}_{+}(E)$ the bundle over $X$ whose fiber over any point $x \in X$ is equal to $\mathbb{P}_{+}\left(E_{x}\right)$ (where $E_{x}$ denotes the fiber of $E$ over $x)$.

- For a convex compact set $A \in \mathcal{K}(V)$ let us denote by $h_{A}$ the supporting functional of $A, h_{A}: V^{*} \rightarrow \mathbb{R}$. It is defined by

$$
h_{A}(y):=\sup \{y(x) \mid x \in A\} .
$$

- Let $L$ denote the (real) line bundle over $\mathbb{P}_{+}\left(V^{*}\right)$ such that its fiber over an oriented line $l \in \mathbb{P}_{+}\left(V^{*}\right)$ is equal to the dual line $l^{*}$.

- For a smooth vector bundle $E$ over a manifold $X$ and $k$ being a non-negative integer or infinity, let us denote by $C^{k}(X, E)$ the space of $C^{k}$-smooth sections of $E$. We denote by $C_{c}^{k}(X, E)$ the space of $C^{k}$-smooth sections with compact support. Let us denote by $C^{-\infty}(X, E)$ the space of generalized sections of $E$ which is equal by definition to the dual space $\left(C_{c}^{\infty}\left(X, E^{*} \otimes\left|\omega_{X}\right|\right)\right)^{*}$. We have the canonical imbedding $C^{k}(X, E) \hookrightarrow C^{-\infty}(X, E)$ (see e.g. 12, Ch. VI $\S 1)$.

Let $K \in \mathcal{K}(V)$. Let $x \in K$.

1.1.1 Definition. A tangent cone to $K$ at $x$ is the set denoted by $T_{x} K$ which is equal to the closure of the set $\{y \in V \mid \exists \varepsilon>0 x+\varepsilon y \in K\}$.

It is easy to see that $T_{x} K$ is a closed convex cone.

1.1.2 Definition. A normal cone to $K$ at $x$ is the set

$$
\left(T_{x} K\right)^{o}:=\left\{y \in V^{*} \mid y(x) \geq 0 \forall x \in T_{x} K\right\} .
$$

Thus $\left(T_{x} K\right)^{o}$ is also a closed convex cone.

1.1.3 Definition. Let $K \in \mathcal{K}(V)$. The characteristic cycle of $\mathrm{K}$ is the set

$$
C C(K):=\cup_{x \in K}\left(T_{x} K\right)^{o} .
$$

It is easy to see that $C C(K)$ is a closed $n$-dimensional subset of $T^{*} V=V \times V^{*}$ invariant with respect to the multiplication by non-negative numbers acting on the second factor. For some references on the characteristic and normal cycles of various sets see Remark 1.3.1 below. 


\subsection{Subanalytic sets.}

In this subsection we review some basic facts from the theory of subanalytic sets of Hironaka. For more information see [16], [17, [14, [15], 8], 24], and $\S 8.2$ of [18]. Let $X$ be a real analytic manifold.

1.2.1 Definition. Let $Z$ be a subset of the manifold $X . Z$ is called subanalytic at a point $x \in X$ if there exists an open neighborhood $U$ of $x$, compact real analytic manifolds $Y_{j}^{i}, i=$ $1,2, j=1, \ldots, N$, and real analytic maps

$$
f_{j}^{i}: Y_{j}^{i} \rightarrow X
$$

such that

$$
Z \cap U=U \cap \cup_{j=1}^{N}\left(f_{j}^{1}\left(Y_{j}^{1}\right) \backslash f_{j}^{2}\left(Y_{j}^{2}\right)\right) .
$$

$Z$ is called subanalytic in $X$ if $Z$ is subanalytic at every point of $X$.

1.2.2 Proposition. (i) Let $Z$ be a subanalytic subset of the manifold $X$. Then the closure and the interior of $Z$ are subanalytic subsets.

(ii) The connected components of a subanalytic set are locally finite and subanalytic.

(iii) Let $Z_{1}$ and $Z_{2}$ be subanalytic subsets of the manifold $X$. Then $Z_{1} \cup Z_{2}, Z_{1} \cap Z_{2}$, and $Z_{1} \backslash Z_{2}$ are subanalytic.

1.2.3 Definition. Let $Z$ be a subanalytic subset of the manifold $X$. A point $x \in Z$ is called regular if there exists an open neighborhood $U$ of $x$ in $X$ such that $U \cap Z$ is a submanifold of $X$.

The set of regular points is denoted by $Z_{\text {reg }}$. Define the set of singular points by $Z_{\text {sing }}:=$ $Z \backslash Z_{\text {reg }}$.

1.2.4 Proposition. The sets $Z_{\text {reg }}$ and $Z_{\text {sing }}$ are subanalytic, and $Z \subset \bar{Z}_{\text {reg }}$.

If $x \in Z_{\text {reg }}$ then the dimension of $Z$ at $x$ is well defined; it is denoted by $\operatorname{dim}_{x} Z$. Define

$$
\operatorname{dim} Z:=\sup _{x \in Z_{\text {reg }}} \operatorname{dim}_{x}(Z)
$$

Clearly $\operatorname{dim} Z \leq \operatorname{dim} X$.

1.2.5 Proposition. Let $Z \subset X$ be a subanalytic subset. Then

(i) $\operatorname{dim}\left(Z \backslash Z_{\text {reg }}\right)<\operatorname{dim} Z$;

(ii) $\operatorname{dim}(\bar{Z} \backslash Z)<\operatorname{dim} Z$.

1.2.6 Definition ([18], §9.7). An integer valued function $f: X \rightarrow \mathbb{Z}$ is called constructible if

1) for any $m \in \mathbb{Z}$ the set $f^{-1}(m)$ is subanalytic;

2) the family of sets $\left\{f^{-1}(m)\right\}_{m \in \mathbb{Z}}$ is locally finite. 
Clearly the set of constructible $\mathbb{Z}$-valued functions is a ring with pointwise multiplication. As in [18] we denote this ring by $C F(X)$. Define

$$
\mathcal{F}:=C F(X) \otimes_{\mathbb{Z}} \mathbb{C} .
$$

Thus $\mathcal{F}$ is a subalgebra of the $\mathbb{C}$-algebra of complex valued functions on $X$. In the rest of the article the elements of $\mathcal{F}$ will be called constructible functions.

Let $\mathcal{F}_{c}(X)$ denote the subspace of $\mathcal{F}(X)$ of compactly supported constructible functions. Clearly $\mathcal{F}_{c}(X)$ is a subalgebra of $\mathcal{F}(X)$ (without unit if $X$ is non-compact).

For a subset $P \subset X$ let us denote by $\mathbb{1}_{P}$ the indicator function of $P$, namely

$$
\mathbb{1}_{P}(x)=\left\{\begin{array}{lll}
1 & \text { if } & x \in P \\
0 & \text { if } & x \notin P .
\end{array}\right.
$$

1.2.7 Proposition. (i) Any function $f \in \mathcal{F}(X)$ can be presented locally as finite linear combination of functions of the form $\mathbb{1}_{Q}$ where $Q$ is a closed subanalytic subset.

(ii) Any function $f \in \mathcal{F}_{c}(X)$ can be presented as finite linear combination of functions of the form $\mathbb{1}_{Q}$ where $Q$ is a compact subanalytic subset.

Proof. Both statements are proved similarly. Let prove say the second one. Let $f \in$ $\mathcal{F}_{c}(X)$. We prove the statement by the induction on $\operatorname{dim}(\operatorname{supp} f$ ) (note that supp $f$ is a subanalytic subset). If $\operatorname{dim}(\operatorname{supp} f)=0$ then there is nothing to prove. Let us assume that we have proven the results for all constructible functions with the dimension of support strictly less than $k$. Let us prove it for $k$. Clearly $f$ is a finite linear combination of functions of the form $\mathbb{1}_{Q}$ where $Q$ is relatively compact subanalytic subset with $\operatorname{dim} Q \leq k$. But

$$
\mathbb{1}_{Q}=\mathbb{1}_{\bar{Q}}-\mathbb{1}_{\bar{Q} \backslash Q} .
$$

By Proposition 1.2.2 the set $\bar{Q} \backslash Q$ is subanalytic, and by Proposition 1.2.5 (ii) $\operatorname{dim}(\bar{Q} \backslash Q)<k$. The induction assumption implies the result. Q.E.D.

\subsection{Characteristic and normal cycles.}

In Subsection [1.1 we have reminded the notion of characteristic cycle of convex compact sets. In this subsection we remind the notion of characteristic cycle and very similar notion of normal cycles of sets either from the class $\mathcal{P}(X)$ on a smooth manifold $X$, or the class of subanalytic subsets of a real analytic manifold $X$ (in fact in the real analytic situation these notions will be discussed more generally for constructible functions on $X$ following [18). The notions of characteristic and normal cycles of various classes of sets coincide on the pairwise intersections of these classes.

1.3.1 Remark. The notion of the characteristic cycle is not new. First an almost equivalent notion of normal cycle (see below) was introduced by Wintgen [25], and then studied further by Zähle [26] by the tools of geometric measure theory. Characteristic cycles of subanalytic sets of real analytic manifolds were introduced by J. Fu [1] using the tools of geometric measure theory and independently by Kashiwara (see [18, Chapter 9) using the tools of the sheaf theory. J. Fu's article [11] develops a more general approach to define the normal cycle for more general sets than subanalytic or convex ones (see Theorem 3.2 in [1]). Applications of the method of normal cycles to integral geometry can be found in [10]. 
For simplicity of the exposition, in the rest of this subsection we will assume that the manifold $X$ is oriented. Then characteristic (resp. normal) cycle is a cycle in $T^{*} X$ (resp. $\mathbb{P}_{+}\left(T^{*} X\right)$. Nevertheless the characteristic and normal cycles can be defined on non-oriented (even non-orientable) manifolds; then they are cycles taking values is the local system $p^{*} o$ where $o$ is the orientation bundle over $X$ and $p: T^{*} X \rightarrow X$ is the canonical projection. We refer to [18], §9.3, for the details on that. Though in our applications to valuations of these notions we will need the general case of not necessarily orientable manifolds, we will ignore here this subtlety. Thus here we discuss the notions of characteristic and normal cycles for oriented manifolds, but apply it below for general manifolds.

Let us assume first that $X$ is a smooth oriented manifold. Set $n=\operatorname{dim} X$. Let $P \in \mathcal{P}(X)$. For any point $x \in P$ let us define the tangent cone to $P$ at $x$, denoted by $T_{x} P$, the set

$T_{x} P:=\left\{\xi \in T_{x} X \mid\right.$ there exists a $C^{1}-$ map $\gamma:[0,1] \rightarrow P$ such that $\gamma(0)=x$ and $\left.\gamma^{\prime}(0)=\xi\right\}$.

It is easy to see that $T_{x} P$ coincides with the usual tangent space if $x$ is an interior point of $P$. In general $T_{x} P$ is a closed convex polyhedral cone in $T_{x} X$. Define

$$
C C(P):=\cup_{x \in P}\left(T_{x} P\right)^{o}
$$

where for a convex cone $C$ in a linear space $W$ one denotes $C^{o}$ its dual cone in $W^{*}$ :

$$
C^{o}:=\left\{y \in W^{*} \mid y(x) \geq 0 \text { for any } x \in C\right\} .
$$

Clearly $C C(P)$ is invariant under the group $\mathbb{R}_{>0}$ of positive real numbers acting on the cotangent bundle $T^{*} X$ by multiplication along the fibers. It is easy to see that $C C(P)$ is an $n$-dimensional Lagrangian submanifold of $T^{*} X$ with singularities. A choice of orientation on $X$ induces an orientation on $C C(P)$. Then $C C(P)$ becomes a cycle, i.e. $\partial(C C(P))=0$.

Let us assume now that $X$ is a real analytic manifold. Again we assume that $X$ is oriented. Let $C F(X)$ be the ring of integer valued constructible functions as in Definition 1.2.6, and let $\mathcal{F}$ denote the algebra of (complex valued) constructible functions as in (1.2.1).

In 18, $§ 9.7$, there was constructed a group homomorphism, also called characteristic cycle,

$$
C C: C F(X) \rightarrow \mathcal{L}(X)
$$

where $\mathcal{L}(X)$ denotes the group of Lagrangian conic subanalytic cycles (with values in $p^{*} O$ in the non-oriented case). For the formal definitions we refer to [18], $\S \S 9.7,9.2$. Here we describe $\mathcal{L}(X)$ in a somewhat unformal way when $X$ is oriented. An arbitrary element $\lambda \in \mathcal{L}(X)$ is an $n$-cycle on $T^{*} X$ (i.e. $\partial \lambda=0$ ) which locally over $X$ can be written as a finite sum $\lambda=\sum_{j} m_{j}\left[\Lambda_{j}\right]$ where $m_{j}$ are integers, $\Lambda_{j}$ are subanalytic oriented Lagrangian locally closed submanifolds of $T^{*} X$ which are conic, i.e. invariant under the action of the group of positive real numbers $\mathbb{R}_{>0}$ on $T^{*} X$, and $\left[\Lambda_{j}\right]$ denotes the chain class of $\Lambda_{j}$.

Let us summarize some basic properties of $C C$ which will be used later. First $C C$ commutes with restrictions of functions to open subsets of $X$.

Let $P \subset X$ be a compact subanalytic subset. Assume in addition that $P \in \mathcal{P}(X)$. Then $C C\left(\mathbb{1}_{P}\right)$ coincides with the characteristic cycle $C C(P)$ defined above in (1.3.1). Thus for a subanalytic closed subset $Q$ we will also denote by $C C(Q)$ the characteristic cycle $C C\left(\mathbb{1}_{Q}\right)$.

For a (locally closed) submanifold $S \subset X$ let us denote by $T_{S}^{*} X$ the conormal bundle of $S$. If $S$ is subanalytic then $T_{S}^{*} X$ is a subanalytic subset of $T^{*} X$ (Proposition 8.3.1 in [18]). 
1.3.2 Lemma. Let $Q \subset X$ be a relatively compact subanalytic subset. Then the closure $\bar{Q}$ can be presented as a finite union $\bar{Q}=\cup_{j} Q_{j}$ of (locally closed) subanalytic submanifolds such that

$$
\operatorname{supp}\left(C C\left(\mathbb{1}_{Q}\right)\right) \subset \cup_{j} T_{Q_{j}}^{*} X .
$$

Proof. Using induction in $\operatorname{dim} Q$ and Propositions 1.2.4, 1.2.5 we may replace $Q$ by $Q_{\text {reg }}$ and thus assume that $Q$ is a (locally closed) submanifold of $X$.

Let us consider the subanalytic covering $X=Q \sqcup(X \backslash Q)$. By Theorem 8.3.20 of [18] there exists a $\mu$-stratification $X=\bigsqcup_{\beta} X_{\beta}$ which is a refinement of the above covering (for the definition of $\mu$-stratification see Definition 8.3.19 of [18]).

Let us denote by $j: Q \rightarrow X$ the identity imbedding. Let $\mathbb{C}_{Q}$ denote the constant sheaf on $Q$ (with complex coefficients). Let $T_{Q}:=j ! \mathbb{\mathbb { C }}_{Q}$ be the extension of $\underline{\mathbb{C}}_{Q}$ by zero. By the definition of the characteristic cycle $([18, \S 9.7)$

$$
C C\left(\mathbb{1}_{Q}\right)=C C\left(T_{Q}\right)
$$

where in the right hand side stays the characteristic cycle of the sheaf $T_{Q}$ (see $\S 9.4$ of [18]). Note that $T_{Q}$ is obviously constructible with respect to the $\mu$-stratification $\left\{X_{\beta}\right\}$. It follows from the definition of the characteristic cycle of a sheaf that

$$
\operatorname{supp} C C\left(T_{Q}\right) \subset S S\left(T_{Q}\right)
$$

where $S S(\cdot)$ denotes the singular support of a sheaf (see $\S 5.1$ of [18]). Proposition 8.4.1 of [18] implies that $S S\left(T_{Q}\right) \subset \bigsqcup_{\beta} T_{X_{\beta}}^{*} X$. But since $\left.T_{Q}\right|_{X \backslash \bar{Q}}=0$ one has

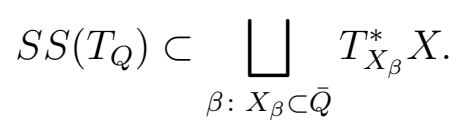

Let us choose the covering $\bar{Q}=\cup_{\alpha} Q_{\alpha}$ where each $Q_{\alpha}$ is equal to one of the sets $X_{\beta}$ contained in $\bar{Q}$. Thus (1.3.2)-(1.3.4) imply

$$
C C(Q) \subset \cup_{\alpha} T_{Q_{\alpha}}^{*} X .
$$

Lemma is proved. Q.E.D.

Let us remind the definition of a normal cycle. We will treat all the cases of subanalytic, convex, $\mathcal{P}(X)$-sets, and constructible functions simultaneously since in all these cases we already have the notion of characteristic cycle.

Let $f$ be an element of one of these families. Let $C C(f)$ be its characteristic cycle. Let us denote by $\underline{C C}(f)$ the intersection of $C C(f)$ with the open subset of $T^{*} X$ obtained by removing the zero section $\underline{0}$. Then $\underline{C C}(f)$ is an $n$-cycle in $T^{*} X \backslash \underline{0}$ invariant under the multiplication by positive real numbers. Let $q: T^{*} X \backslash \underline{0} \rightarrow \mathbb{P}_{+}\left(T^{*} X\right)$ denote the canonical quotient map. (Remind that $\mathbb{P}_{+}\left(T^{*} X\right)$ denotes the bundle over $X$ whose fiber over a point $x \in X$ is equal to the manifold of oriented lines in $T_{x}^{*} X$ passing through the origin.)

It is easy to see that there exists unique $(n-1)$-cycle in $\mathbb{P}_{+}\left(T^{*} X\right)$ denoted by $\tilde{C C}(f)$ such that $C C(f)=q^{-1}(\tilde{C C}(f))$. Consider the (antipodal) involution $a: \mathbb{P}_{+}\left(T^{*} X\right) \rightarrow \mathbb{P}_{+}\left(T^{*} X\right)$ changing the orientation of each line. Then by definition the normal cycle $N(f)$ is equal to $a_{*}(\tilde{C C}(f))$. It is easy to see that if $C C(f)$ is a subanalytic cycle then $N(f)$ is a subanalytic cycle, in particular if $f$ is a constructible function then $N(f)$ is a subanalytic cycle. Also it is known that $N(f)$ is a Legendrian cycle when $\mathbb{P}_{+}\left(T^{*} X\right)$ is equipped with the canonical contact structure. 


\subsection{Some valuation theory.}

First let us remind some results from [1]. Let $V$ be an $n$-dimensional real vector space. Let $\bar{K}=\left(K_{1}, K_{2}, \ldots, K_{s}\right)$ be an $s$-tuple of compact convex subsets of $V$. Let $r \in \mathbb{N} \cup\{\infty\}$. For any $\mu \in C^{r}\left(V,\left|\omega_{V}\right|\right)$ consider the function $M_{\bar{K}} F: \mathbb{R}_{+}^{s} \rightarrow \mathbb{C}$, where $\mathbb{R}_{+}^{s}=\left\{\left(\lambda_{1}, \ldots, \lambda_{s}\right) \mid \lambda_{j} \geq\right.$ $0\}$, defined by

$$
\left(M_{\bar{K}} \mu\right)\left(\lambda_{1}, \ldots, \lambda_{s}\right)=\mu\left(\sum_{i=1}^{s} \lambda_{i} K_{i}\right) .
$$

1.4.1 Theorem $([\mathbf{1}])$. (1) $M_{\bar{K}} \mu \in C^{r}\left(\mathbb{R}_{+}^{s}\right)$ and $M_{\bar{K}}$ is a continuous operator from $C^{r}\left(V,\left|\omega_{V}\right|\right)$ to $C^{r}\left(\mathbb{R}_{+}^{s}\right)$.

(2) Assume that a sequence $\mu^{(m)}$ converges to $\mu$ in $C^{r}\left(V,\left|\omega_{V}\right|\right)$. Let $K_{i}^{(m)}, K_{i}, i=$ $1, \ldots, s, m \in \mathbb{N}$, be convex compact sets in $V$, and for every $i=1, \ldots, s K_{i}^{(m)} \rightarrow K_{i}$ in the Hausdorff metric as $m \rightarrow \infty$. Then $M_{\bar{K}^{(m)}} \mu^{(m)} \rightarrow M_{\bar{K}} \mu$ in $C^{r}\left(\mathbb{R}_{+}^{s}\right)$ as $m \rightarrow \infty$.

1.4.2 Definition. a) A function $\phi: \mathcal{K}(V) \rightarrow \mathbb{C}$ is called a valuation if for any $K_{1}, K_{2} \in \mathcal{K}(V)$ such that their union is also convex one has

$$
\phi\left(K_{1} \cup K_{2}\right)=\phi\left(K_{1}\right)+\phi\left(K_{2}\right)-\phi\left(K_{1} \cap K_{2}\right) .
$$

b) A valuation $\phi$ is called continuous if it is continuous with respect to the Hausdorff metric on $\mathcal{K}(V)$.

For the classical theory of valuations we refer to the surveys McMullen-Schneider [21. and McMullen 20]. For the general background from convexity we refer to Schneider [23].

In [4] one has introduced a class $S V(V)$ of valuations called smooth valuations. We refer to [4] for an axiomatic definition. Here we only mention that $S V(V)$ is a $\mathbb{C}$-linear space (with the obvious operations) with a natural Fréchet topology. In this article we will need a description of $S V(V)$ which is Theorem 1.4.3 below.

Let us denote by ${ }^{\mathbf{C}} L$ the (complex) line bundle over $\mathbb{P}_{+}\left(V^{*}\right)$ whose fiber over $l \in \mathbb{P}_{+}\left(V^{*}\right)$ is equal to $l^{*} \otimes_{\mathbb{R}} \mathbb{C}$ (where $l^{*}$ denotes the dual space to $l$ ).

Note that for any convex compact set $A \in \mathcal{K}(V)$ the supporting functional $h_{A}$ is a continuous section of ${ }^{\mathbf{C}} L$, i.e $h_{A} \in C\left(\mathbb{P}_{+}\left(V^{*}\right),{ }^{\mathbf{C}} L\right)$.

1.4.3 Theorem ([4], Corollary 3.1.7). There exists a continuous linear map

$$
\mathcal{T}: \oplus_{k=0}^{n} C^{\infty}\left(V \times \mathbb{P}_{+}\left(V^{*}\right)^{k},\left|\omega_{V}\right| \otimes^{C} L^{\otimes k}\right) \rightarrow S V(V)
$$

which is uniquely characterized by the following property: for any $k=0,1, \ldots, n$, any $\mu \in$ $C^{\infty}\left(V,\left|\omega_{V}\right|\right)$, any strictly convex compact sets $A_{1}, \ldots, A_{k}$ with smooth boundaries, and any $K \in \mathcal{K}(V)$ one has

$$
\mathcal{T}\left(\mu \otimes h_{A_{1}} \otimes \cdots \otimes h_{A_{k}}\right)(K)=\frac{\partial^{k}}{\partial \lambda_{1} \ldots \partial \lambda_{k}} \mid{ }_{0} \mu\left(K+\sum_{i=1}^{k} \lambda_{i} A_{i}\right)
$$

where $\lambda_{i} \geq 0$ in the right hand side.

Moreover the map $\mathcal{T}$ is an epimorphism. 
In [5] one has introduced for any smooth manifold $X$ a class of finitely additive measures on the family of simple subpolyhedra $\mathcal{P}(X)$. This class is denoted by $V^{\infty}(X)$. It is a $\mathbb{C}$ linear space (with the obvious operations). Then $V^{\infty}(X)$ has a natural Fréchet topology. Moreover in the case of linear Fréchet space $V$ any element $\phi \in V^{\infty}(V)$ being restricted to $\mathcal{K}(V) \cap \mathcal{P}(V)$ has a (unique) extension by continuity in the Hausdorff metric to $\mathcal{K}(V)$, and this extension belongs to $S V(V)$. Thus one gets a linear map

$$
V^{\infty}(V) \rightarrow S V(V)
$$

In [5], Proposition 2.4.10, the following result was proved.

1.4.4 Proposition. The above constructed map $V^{\infty}(V) \rightarrow S V(V)$ is an isomorphism of Fréchet spaces.

We will also need the following description of $V^{\infty}(X)$ obtained in [5] (based on some results on normal cycles from Section 2 of [6]). Let us denote by $T^{*} X$ the cotangent bundle of $X$. Let $p: T^{*} X \rightarrow X$ be the canonical projection. Let $\Omega^{n}$ denote the vector bundle of $n$-forms over $T^{*} X$. Let us denote by $o$ the orientation bundle over $X$. Let us denote by $\tilde{C}^{\infty}\left(T^{*} X, \Omega^{n} \otimes p^{*} o\right)$ the space of $C^{\infty}$-smooth sections of the bundle $\Omega^{n} \otimes p^{*} o$ such that the restriction of the projection $p$ to the support of such section is a proper map.

1.4.5 Theorem ([5], Theorem 0.1.3). (i) Let $\omega \in \tilde{C}^{\infty}\left(T^{*} X, \Omega^{n} \otimes p^{*} o\right)$. The functional $\mathcal{P}(X) \rightarrow \mathbb{C}$

$$
P \mapsto \int_{C C(P)} \omega
$$

is a smooth valuation.

(ii) Conversely, any smooth valuation $\phi \in V^{\infty}(X)$ has the form 1.4.1), i.e. there exists a form $\omega \in \tilde{C}^{\infty}\left(T^{*} X, \Omega^{n} \otimes p^{*} o\right)$ such that $\phi(P)=\int_{C C(P)} \omega$ for any $P \in \mathcal{P}(X)$.

1.4.6 Remark. (1) The integration (1.4.1) is well defined since a choice of orientation of the manifold $X$ induces an orientation of $C C(P)$.

(2) A presentation of a valuation $\phi$ in the form (1.4.1) is highly non-unique.

Let us describe the multiplicative structure on $V^{\infty}(X)$ following [6]. It was shown in [5] that the assignment to any open subset $U \subset X$

$$
U \mapsto V^{\infty}(U)
$$

with the natural restriction maps is a sheaf. The product on smooth valuations commutes with the restrictions to open subsets. Hence it is enough to describe the product locally, say under the assumption that $X$ is diffeomorphic to $\mathbb{R}^{n}$. Let us fix a diffeomorphism $X \stackrel{\sim}{\rightarrow} \mathbb{R}^{n}$. Proposition 1.4.4 provides an isomorphism $V^{\infty}\left(\mathbb{R}^{n}\right) \stackrel{\sim}{\rightarrow} S V\left(\mathbb{R}^{n}\right)$. In [4 the author has described the product on $S V\left(\mathbb{R}^{n}\right)$ which we will remind below. The main point of [6] was to show that the obtained product on $V^{\infty}(X)$ does not depend on the choices of diffeomorphisms. 
Thus it remains to describe the product on $S V\left(\mathbb{R}^{n}\right)$ following [4]. The product

$$
S V\left(\mathbb{R}^{n}\right) \times S V\left(\mathbb{R}^{n}\right) \rightarrow S V\left(\mathbb{R}^{n}\right)
$$

is a continuous map which is uniquely defined by the distributivity and the following property: let $\phi, \psi \in S V\left(\mathbb{R}^{n}\right)$ have the form

$$
\begin{gathered}
\phi(K)=\left.\frac{\partial^{k}}{\partial \lambda_{1} \ldots \partial \lambda_{k}}\right|_{0} \mu\left(K+\sum_{i=1}^{k} \lambda_{i} A_{i}\right), \\
\psi(K)=\left.\frac{\partial^{l}}{\partial \mu_{1} \ldots \partial \mu_{l}}\right|_{0} \mu\left(K+\sum_{j=1}^{l} \mu_{j} B_{j}\right)
\end{gathered}
$$

where $0 \leq k, l \leq n ; \mu, \nu$ are smooth densities on $\mathbb{R}^{n} ; A_{1}, \ldots, A_{k}, B_{1}, \ldots, B_{l}$ are strictly convex compact sets with smooth boundaries, $K$ is an arbitrary convex compact subset in $\mathbb{R}^{n}$. Then

$$
(\phi \cdot \psi)(K)=\left.\frac{\partial^{k+l}}{\partial \lambda_{1} \ldots \partial \lambda_{k} \partial \mu_{1} \ldots \partial \mu_{l}}\right|_{0}(\mu \otimes \nu)\left(\Delta(K)+\left(\left(\sum_{i=1}^{k} \lambda_{i} A_{i}\right) \times\left(\sum_{j=1}^{l} \mu_{j} B_{j}\right)\right)\right)
$$

where $\Delta: \mathbb{R}^{n} \hookrightarrow \mathbb{R}^{n} \times \mathbb{R}^{n}$ is the diagonal imbedding, $\mu \otimes \nu$ denotes the usual exterior product of densities. Note that in (1.4.2)-(1.4.4) the derivatives exist due to Theorem 1.4.1.

Equipped with this product, the space $V^{\infty}(X)$ becomes a commutative associative algebra with unit (the unit is the Euler characteristic).

Let us describe the Euler-Verdier involution $\sigma$ on $V^{\infty}(X)$ following [5]. Let $a: T^{*} X \rightarrow$ $T^{*} X$ be the involution of multiplication by -1 in each fiber of the projection $p: T^{*} X \rightarrow X$. It induces the involution

$$
a^{*}: \tilde{C}^{\infty}\left(T^{*} X, \Omega^{n} \otimes p^{*} o\right) \rightarrow \tilde{C}^{\infty}\left(T^{*} X, \Omega^{n} \otimes p^{*} o\right) .
$$

We have the following proposition.

1.4.7 Proposition ([5], Proposition 3.3.1). The involution $(-1)^{n} a^{*}$ factorizes (uniquely) to the involution of $V^{\infty}(X)$ which is denoted by $\sigma$. Moreover $\sigma$ commutes with the restrictions to open subsets and thus induces an involution of the sheaf $\mathcal{V}_{X}^{\infty}$ which is also denoted by $\sigma$.

\subsection{Filtration on valuations.}

In [5] we have introduced on the space of smooth valuations $V^{\infty}(X)$ a canonical finite filtration by closed subspaces:

$$
V^{\infty}(X)=W_{0}(X) \supset W_{1}(X) \supset \cdots \supset W_{n}(X)
$$

where $n=\operatorname{dim} X$. Let us remind some of the main properties of this filtration.

1.5.1 Proposition ([5], Proposition 3.1.2). The assignment to each open subset $U \subset X$

$$
U \mapsto W_{i}(U)
$$

is a subsheaf of $\mathcal{V}_{X}^{\infty}$. (This sheaf is denoted by $\mathcal{W}_{i}$.) 
It turns out that the associated graded sheaf $g r_{\mathcal{W}} \mathcal{V}_{X}^{\infty}:=\bigoplus_{i=0}^{n} \mathcal{W}_{i} / \mathcal{W}_{i+1}$ admits a simple description in terms of translation invariant valuations. To state it let us denote by $\operatorname{Val}(T X)$ the (infinite dimensional) vector bundle over $X$ such that its fiber over a point $x \in X$ is equal to the space $\operatorname{Val}^{s m}\left(T_{x} X\right)$ of smooth translation invariant valuations on the tangent space $T_{x} X$. By McMullen's theorem [19] the space $\operatorname{Val}^{s m}\left(T_{x} X\right)$ has natural grading by the degree of homogeneity which must be an integer between 0 and $n$. Thus $\operatorname{Val}(T X)$ is a graded vector bundle. Let us denote by $\underline{\operatorname{Val}}(T X)$ the sheaf $U \mapsto C^{\infty}(U, \operatorname{Val}(T X))$ where the last space denotes the space of infinitely smooth sections of $\operatorname{Val}(T X)$ over $U$.

1.5.2 Theorem ([5], Theorem 0.1.2 and Section 3). There exists a canonical isomorphism of graded sheaves

$$
g r_{\mathcal{W}} \mathcal{V}_{X}^{\infty} \simeq \underline{\operatorname{Val}}(T X)
$$

Moreover for any open subset $U \subset X$ the induced isomorphism on global sections is isomorphism of linear topological spaces.

This theorem provides a description of smooth valuations since translation invariant valuations are studied much better.

1.5.3 Remark. Interpreted appropriately, Theorem 1.5.2 says in particular that the last term of the filtration $\mathcal{W}_{n}$ is canonically isomorphic to the sheaf of $C^{\infty}$-smooth measures (=densities) on $X$, and the first quotient $\mathcal{V}_{X}^{\infty} / \mathcal{W}_{1}$ is canonically isomorphic to the sheaf of $C^{\infty}$-smooth functions on $X$.

The filtration $\left\{W_{\bullet}\right\}$ on valuations can be interpreted in terms of Theorem 1.4.5 as follows. First remind the general construction of a filtration differential forms on a total space of a bundle.

Let $X$ be a smooth manifold. Let $p: P \rightarrow X$ be a smooth bundle. Let $\Omega^{N}(P)$ be the vector bundle over $P$ of $N$-forms. For a vector space $R$ we denote by $G r_{N}(R)$ the Grassmannian of $N$-dimensional linear subspaces in $R$. Let us introduce a filtration of $\Omega^{N}(P)$ by vector subbundles $W_{i}(P)$ as follows. For every $y \in P$ set

$$
\begin{aligned}
\left(W_{i}(P)\right)_{y}:=\left\{\omega \in \wedge^{N} T_{y}^{*} P|\omega|_{F} \equiv 0 \text { for all } F \in G r_{N}\left(T_{y} P\right)\right. & \\
& \text { with } \left.\operatorname{dim}\left(F \cap T_{y}\left(p^{-1} p(y)\right)\right)>N-i\right\} .
\end{aligned}
$$

Clearly we have

$$
\Omega^{N}(P)=W_{0}(P) \supset W_{1}(P) \supset \cdots \supset W_{N}(P) \supset W_{N+1}(P)=0 .
$$

Let us discuss this filtration in greater detail following 4 .

Let us make some elementary observations from linear algebra. Let $L$ be a finite dimensional vector space. Let $E \subset L$ be a linear subspace. For a non-negative integer $i$ set

$$
W(L, E)_{i}:=\left\{\omega \in \wedge^{N} L^{*}|\omega|_{F} \equiv 0 \text { for all } F \subset L \text { with } \operatorname{dim}(F \cap E)>N-i\right\} .
$$

Clearly

$$
\wedge^{N} L^{*}=W(L, E)_{0} \supset W(L, E)_{1} \supset \cdots \supset W(L, E)_{N} \supset W(L, E)_{N+1}=0 .
$$


1.5.4 Lemma ([4], Lemma 5.2.3). There exists canonical isomorphism of vector spaces

$$
W(L, E)_{i} / W(L, E)_{i+1}=\wedge^{N-i} E^{*} \otimes \wedge^{i}(L / E)^{*} .
$$

Let us apply this construction in the context of integration with respect to the characteristic cycle. Let $X$ be a smooth manifold of dimension $n$. Let $P:=T^{*} X$ be the cotangent bundle. Let $p: P \rightarrow X$ be the canonical projection. Let us denote by $o$ the orientation bundle on $X$. The above construction gives a filtration of $\Omega^{n}(P)$ by subbundles

$$
\Omega^{n}(P)=W_{0}\left(\Omega^{n}(P)\right) \supset \cdots \supset W_{n}\left(\Omega^{n}(P)\right) .
$$

Twisting this filtration by $p^{*} o$ we get a filtration of $\Omega^{n}(P) \otimes p^{*} o$ by subbundles denoted by $W_{i}\left(\Omega^{n}(P) \otimes p^{*} o\right)$.

Let us denote by $\tilde{C}^{\infty}\left(P, W_{i}\left(\Omega^{n} \otimes p^{*} o\right)\right)$ the space of infinitely smooth sections of the bundle $W_{i}\left(\Omega^{n} \otimes p^{*} o\right)$ such that the restriction of the projection $p$ to the support of these sections is proper. The we have the following result.

1.5.5 Theorem ([5], Proposition 3.1.9). For any valuation $\phi \in W_{i}(X)$ there exists $\omega \in \tilde{C}^{\infty}\left(T^{*} X, W_{i}\left(\Omega^{n} \otimes p^{*} o\right)\right)$ such that for any $P \in \mathcal{P}(X)$

$$
\phi(P)=\int_{C C(P)} \omega .
$$

Conversely any such valuation belongs to $W_{i}(X)$.

\section{A technical lemma.}

In this section we will prove a technical lemma which will be used later on in this article.

2.1.1 Lemma. Let $i=0,1, \ldots, n$. Let $\phi \in W_{i}\left(V_{c}^{\infty}(X)\right)$. Then there exists a compactly supported form $\omega \in C_{c}^{\infty}\left(T^{*} X, W_{i}\left(\Omega^{n} \otimes p^{*} o\right)\right)$ such that

$$
\phi(P)=\int_{C C(P)} \omega \text { for any } P \in \mathcal{P}(X) .
$$

2.1.2 Remark. A version of this lemma for smooth valuations without the assumption on the compactness of support was proved in [5], Proposition 3.1.9; it will be used in the proof of Lemma 2 .

Proof of Lemma 2] As in [5] consider the sheaves on $X$

$$
\begin{array}{r}
\mathcal{W}_{i}(U)=W_{i}\left(V^{\infty}(U)\right), \\
\mathcal{W}_{i}^{\prime}(U)=\tilde{C}^{\infty}\left(T^{*} U, W_{i}\left(\Omega^{n} \otimes p^{*} o\right)\right)
\end{array}
$$

for any open subset $U \subset X$; in equality (2.1.3) the symbol $\tilde{C}^{\infty}$ denotes the space of infinitely smooth sections of a vector bundle over $T^{*} U$ such that the restriction of the canonical 
projection $p: T^{*} U \rightarrow U$ to the support of such sections is proper. We have the obvious inclusions:

$$
\begin{aligned}
\mathcal{W}_{n}^{\prime} \subset \mathcal{W}_{n-1}^{\prime} \subset \cdots \subset \mathcal{W}_{0}^{\prime} \\
\mathcal{W}_{n} \subset \mathcal{W}_{n-1} \subset \cdots \subset \mathcal{W}_{0}=\mathcal{V}_{X}^{\infty}
\end{aligned}
$$

The integration over the the characteristic cycle gives a morphism of sheaves

$$
T_{i}: \mathcal{W}_{i}^{\prime} \rightarrow \mathcal{W}_{i}
$$

By Proposition 3.1.9 of [5] $T_{i}$ is an epimorphism of sheaves. Clearly the restriction of $T_{i}$ to $\mathcal{W}_{i+1}^{\prime}$ is equal to $T_{i+1}$. Define the sheaves

$$
\mathcal{K}_{i}:=\operatorname{Ker} T_{i}
$$

We obviously have

$$
\mathcal{K}_{n} \subset \mathcal{K}_{n-1} \subset \cdots \subset \mathcal{K}_{0} \subset \mathcal{W}_{0}^{\prime}
$$

Let us consider the associated graded sheaves

$$
\begin{aligned}
\mathcal{F} & :=\oplus_{i=o}^{n} \mathcal{W}_{i} / \mathcal{W}_{i+1}, \\
\mathcal{F}^{\prime} & :=\oplus_{i=0}^{n} \mathcal{W}_{i}^{\prime} / \mathcal{W}_{i+1}^{\prime} .
\end{aligned}
$$

The epimorphism $T_{0}: \mathcal{W}_{0}^{\prime} \rightarrow \mathcal{W}_{0}$ induces the epimorphism

$$
T: \mathcal{F}^{\prime} \rightarrow \mathcal{F} \text {. }
$$

Define $\mathcal{T}:=\operatorname{Ker} T$. Clearly

$$
\mathcal{T}=\oplus_{i=0}^{n} \mathcal{K}_{i} / \mathcal{K}_{i+1}
$$

Let us denote by $\mathcal{O}_{X}$ the sheaf of $C^{\infty}$-smooth functions on $X$. It was shown in [5] (see the proof of Proposition 3.1.9) that $\mathcal{T}$ is naturally isomorphic to a sheaf of $\mathcal{O}_{X}$-modules. Hence by Section 3.7 of Ch. II in Godement's book [13] one has

$$
H_{c}^{j}(X, \mathcal{T})=0 \text { for } j>0 .
$$

Hence $H_{c}^{j}\left(X, \mathcal{K}_{i} / \mathcal{K}_{i+1}\right)=0$ for $j>0, i=0,1, \ldots, n$. By the long exact sequence we deduce

$$
H_{c}^{j}\left(X, \mathcal{K}_{i}\right)=0 \text { for } j>0, i=0,1, \ldots, n .
$$

For the short exact sequence of sheaves

$$
0 \rightarrow \mathcal{K}_{i} \rightarrow \mathcal{W}_{i}^{\prime} \stackrel{T_{i}}{\rightarrow} \mathcal{W}_{i} \rightarrow 0
$$

consider the beginning of the long exact sequence in cohomology with compact support

$$
H_{c}^{0}\left(X, \mathcal{W}_{i}^{\prime}\right) \rightarrow H_{c}^{0}\left(X, \mathcal{W}_{i}\right) \rightarrow H_{c}^{1}\left(X, \mathcal{K}_{i}\right)
$$

But the last space in (2.1.11) vanishes due to (2.1.10). Hence the map $H_{c}^{0}\left(X, \mathcal{W}_{i}^{\prime}\right) \rightarrow$ $H_{c}^{0}\left(X, \mathcal{W}_{i}\right)$ is surjective. But

$$
\begin{array}{r}
H_{c}^{0}\left(X, \mathcal{W}_{i}\right)=W_{i}\left(V_{c}^{\infty}(X)\right) ; \\
H_{c}^{0}\left(X, \mathcal{W}_{i}^{\prime}\right)=C_{0}^{\infty}\left(T^{*} X, \Omega^{n} \otimes p^{*} o\right) .
\end{array}
$$

Thus lemma is proved. Q.E.D. 


\section{Compatibility of the filtration with the product.}

The main results of this section are Theorems 3.1.1 and 3.1.2 below.

Remind that in Subsection [1.5 we have discussed the canonical filtration by closed subspaces

$$
V^{\infty}(X)=W_{0}(X) \supset W_{1}(X) \supset \cdots \supset W_{n}(X) .
$$

It will be convenient to extend this filtration infinitely by putting

$$
W_{i}(X)=0 \text { for } i>n \text {. }
$$

3.1.1 Theorem. For any $i, j \geq 0$ one has

$$
W_{i}(X) \cdot W_{j}(X) \subset W_{i+j}(X) .
$$

Proof. By Corollary 4.1.4 of [6] $\mathcal{V}_{X}^{\infty}$ is a sheaf of algebras, i.e. the product commutes with the restriction to open subsets. Hence we may assume that $X$ is diffeomorphic to $\mathbb{R}^{n}$. Let us fix a diffeomorphism $X \stackrel{\sim}{\rightarrow} \mathbb{R}^{n}$. Let us consider the induced isomorphism of linear topological spaces

$$
V^{\infty}(X) \stackrel{\sim}{\rightarrow} S V\left(\mathbb{R}^{n}\right)
$$

from Proposition 1.4.4. By Proposition 3.1.3 of [5] the subspace $W_{i}(X)$ is isomorphic under this isomorphism to a closed subspace of $S V\left(\mathbb{R}^{n}\right)$. Let us denote this subspace by $\hat{W}_{i}$; it was explicitly defined in Section 3 of [4] in slightly different notation. Moreover by Theorem 4.1.2(4) of [4]

$$
\hat{W}_{i} \cdot \hat{W}_{j} \subset \hat{W}_{i+j}
$$

Hence our result follows from (3.1.1) and the construction of the product on $V^{\infty}(X)$ described in Subsection 1.4. Q.E.D.

Remind that by Theorem 1.5.2 there exists a canonical isomorphism of graded linear topological spaces

$$
g r_{W} V^{\infty}(X):=\oplus_{i=0}^{n} W_{i}(X) / W_{i+1}(X) \stackrel{\sim}{\rightarrow} C^{\infty}(X, \operatorname{Val}(T X))
$$

where the vector bundle $\operatorname{Val}(T X)$ over $X$ was defined in Subsection 1.5

Observe that $g r_{W} V^{\infty}(X)$ is a graded algebra with the product induced from $V^{\infty}(X)$. Note also that $C^{\infty}(X, \operatorname{Val}(T X))$ is also a graded algebra with the product defined pointwise. Namely if $f, g \in C^{\infty}(X, \operatorname{Val}(T X))$ then for any point $x \in X$

$$
(f \cdot g)(x)=f(x) \cdot g(x) \in \operatorname{Val}^{s m}\left(T_{x} X\right) .
$$

We are going to prove

3.1.2 Theorem. The isomorphism (3.1.2) is an isomorphism of algebras.

Proof. As in the proof of Theorem 3.1.1, the statement is local. Thus we may assume that $X$ is diffeomorphic to $\mathbb{R}^{n}$. Now the result follows from the construction of the product described in Subsection 1.4 and Theorem 4.1.3 of [4] where the corresponding statement was proved for valuations on convex subsets of a linear space. Q.E.D. 


\section{The automorphism property of the Euler-Verdier in- volution.}

The main result of this section is Theorem 4.1.4,

4.1.1 Lemma. Let $\phi \in V^{\infty}(X)$. Let $P \in \mathcal{P}(X)$. Then

$$
(\sigma \phi)(P)=(-1)^{\operatorname{dim} P}(\phi(P)-\phi(\partial P)) .
$$

Proof. Equality (15) in [5] says that for any $\omega \in \tilde{C}^{\infty}\left(T^{*} X, \Omega^{n} \otimes p^{*} o\right)$ and any $P \in \mathcal{P}(X)$ one has

$$
\int_{C C(P)} a^{*} \omega=(-1)^{n-\operatorname{dim} P}\left(\int_{C C(P)} \omega-\int_{C C(\partial P)} \omega\right)
$$

where $\partial P:=P \backslash$ int $P$ and int $P$ denotes the relative interior of $P$. The result follows immediately from Proposition 1.4.7 and (4.1.1). Q.E.D.

From Lemma 4.1.1 we immediately deduce that the Euler-Verdier involution commutes with restriction to submanifolds. More precisely we have the following lemma.

4.1.2 Lemma. Let $Y$ be a smooth submanifold of a manifold $X$. Let $\phi \in V^{\infty}(X)$. Then

$$
\left.(\sigma \phi)\right|_{Y}=\sigma\left(\left.\phi\right|_{Y}\right)
$$

4.1.3 Lemma. Let $\phi \in V^{\infty}\left(\mathbb{R}^{n}\right)$ be a smooth valuation such that for any $K \in \mathcal{K}\left(\mathbb{R}^{n}\right)$ one has

$$
\phi(K)=\left.\frac{\partial^{k}}{\partial \lambda_{1} \ldots \partial \lambda_{k}}\right|_{0} \mu\left(K+\sum_{i=1}^{k} \lambda_{i} A_{i}\right)
$$

where $\mu$ is a smooth density on $\mathbb{R}^{n}$, and $A_{1}, \ldots, A_{k}$ are strictly convex compact subsets with smooth boundaries and containing the origin in the interior. Then

$$
(\sigma \phi)(K)=(-1)^{n-k} \frac{\partial^{k}}{\partial \lambda_{1} \ldots \partial \lambda_{k}} \mid{ }_{0} \mu\left(K+\sum_{i=1}^{k} \lambda_{i}\left(-A_{i}\right)\right) .
$$

Proof. For $k=0$ the lemma is obvious. Let us assume that $k>0$. It is enough to prove (4.1.2) under the assumption that $K$ has non-empty interior and strictly convex smooth boundary. For any $\lambda_{1}, \ldots, \lambda_{k}>0$ the map

$$
\Xi_{\lambda_{1} \ldots \lambda_{k}}: V \times \mathbb{P}_{+}\left(V^{*}\right) \times(0,1] \rightarrow V
$$

given by $(p, n, t) \mapsto p+t \sum_{i=1}^{k} \lambda_{i} \nabla h_{A_{i}}(n)$ induces a homeomorphism of $N(K) \times(0,1]$ onto its image $\left(K+\sum_{i=1}^{k} \lambda_{i} A_{i}\right) \backslash K$ (this is well known; see e.g. Proposition 3.1.2 of [6] where this statement is proved under some more general assumptions). Hence

$$
\phi(K)=\left.\frac{\partial^{k}}{\partial \lambda_{1} \ldots \partial \lambda_{k}}\right|_{0} \int_{N(K) \times[0,1]} \Xi_{\lambda_{1} \ldots \lambda_{k}}^{*} \mu .
$$


Let us denote by $\tilde{a}: \mathbb{P}_{+}\left(V^{*}\right) \rightarrow \mathbb{P}_{+}\left(V^{*}\right)$ the involution of changing an orientation of a line. Then

$$
(\sigma \phi)(K)=\left.(-1)^{n} \frac{\partial^{k}}{\partial \lambda_{1} \ldots \partial \lambda_{k}}\right|_{0} \tilde{a}^{*}\left(\Xi_{\lambda_{1} \ldots \lambda_{k}}^{*} \mu\right)=\left.(-1)^{n} \frac{\partial^{k}}{\partial \lambda_{1} \ldots \partial \lambda_{k}}\right|_{0}\left(\left(\Xi_{\lambda_{1} \ldots \lambda_{k}} \circ \tilde{a}\right)^{*} \mu\right) .
$$

Observe that

$$
\left(\Xi_{\lambda_{1} \ldots \lambda_{k}} \circ a\right)(p, n, t)=p+t \sum_{i=1}^{k} \lambda_{i}\left(\nabla h_{A_{i}}\right)(-n) .
$$

But $h_{-A}(n)=h_{A}(-n)$. Hence

$$
\left(\Xi_{\lambda_{1} \ldots \lambda_{k}} \circ a\right)(p, n, t)=p-t \sum_{i=1}^{k} \lambda_{i}\left(\nabla h_{-A_{i}}\right)(n) .
$$

Note that

$$
\left.\frac{\partial^{k}}{\partial \lambda_{1} \ldots \partial \lambda_{k}}\right|_{0} \mu\left(K+\sum_{i=1}^{k} \lambda_{i}\left(-A_{i}\right)\right)=\left.\frac{\partial^{k}}{\partial \lambda_{1} \ldots \partial \lambda_{k}}\right|_{0} \int_{N(K) \times[0,1]} \tilde{\Xi}_{\lambda_{1} \ldots \lambda_{k}} \mu
$$

where $\tilde{\Xi}_{\lambda_{1} \ldots \lambda_{k}}: V \times \mathbb{P}_{+}\left(V^{*}\right) \times[0,1] \rightarrow V$ is defined by $\tilde{\Xi}_{\lambda_{1} \ldots \lambda_{k}}(p, n, t)=p+t \sum_{i=1}^{k} \lambda_{i} \nabla h_{-A_{i}}(n)$. Now Lemma 4.1 .3 follows from (4.1.3), (4.1.4), (4.1.5). Q.E.D.

4.1.4 Theorem. The Euler-Verdier involution $\sigma: \mathcal{V}_{X}^{\infty} \rightarrow \mathcal{V}_{X}^{\infty}$ is an algebra automorphism. Moreover it preserves the filtration $\mathcal{W}_{\bullet}$, namely $\sigma\left(\mathcal{W}_{i}\right)=\mathcal{W}_{i}$ for any $i=0, \ldots, n$.

Proof. The second part of the theorem was proved in [5]. Thus it remains to show that $\sigma$ is an algebra automorphism. The statement is local thus we may and will assume that $X=\mathbb{R}^{n}$. Let $\phi, \psi \in V^{\infty}\left(\mathbb{R}^{n}\right)$. We may assume that for any $K \in \mathcal{K}\left(\mathbb{R}^{n}\right)$

$$
\phi(K)=\left.\frac{d^{k}}{d \varepsilon^{k}}\right|_{0} \mu(K+\varepsilon A), \psi(K)=\left.\frac{d^{l}}{d \delta^{l}}\right|_{0} \nu(K+\delta B)
$$

where $\mu, \nu$ are smooth densities on $\mathbb{R}^{n}$, and $A, B$ are strictly convex compact subsets with smooth boundaries and containing the origin the interior. Then

$$
(\phi \cdot \psi)(K)=\left.\frac{\partial^{k+l}}{\partial^{k} \varepsilon \cdot \partial^{l} \delta}\right|_{0}(\mu \otimes \nu)(\Delta(K)+(\varepsilon A, \delta B))
$$

where $\Delta: \mathbb{R}^{n} \hookrightarrow \mathbb{R}^{n} \times \mathbb{R}^{n}$ is the diagonal imbedding. By Lemma 4.1.3 one has

$$
\begin{array}{r}
(\sigma \phi)(K)=\left.(-1)^{n-k} \frac{d^{k}}{d \varepsilon^{k}}\right|_{0} \mu(K+\varepsilon(-A)),(\sigma \psi)(K)=\left.(-1)^{n-l} \frac{d^{l}}{d \delta^{l}}\right|_{0} \nu(K+\delta(-B)) \\
(\sigma(\phi \cdot \psi))(K)=\left.(-1)^{2 n-(k+l)} \frac{\partial^{k+l}}{\partial^{k} \varepsilon \cdot \partial^{l} \delta}\right|_{0}(\mu \otimes \nu)(\Delta(K)+(\varepsilon(-A), \delta(-B))) .
\end{array}
$$

Hence we have

$(\sigma \phi \cdot \sigma \psi)(K)=\left.(-1)^{2 n-(k+l)} \frac{\partial^{k+l}}{\partial^{k} \varepsilon \cdot \partial^{l} \delta}\right|_{0}(\mu \otimes \nu)(\Delta(K)+(\varepsilon(-A), \delta(-B)))=(\sigma(\phi \cdot \psi))(K)$.

Q.E.D. 


\section{The integration functional on valuations.}

In Subsection 5.1 we describe canonical linear topology on the space $V_{c}^{\infty}(X)$ of compactly supported smooth valuations. In Subsection 5.2 we construct a canonical continuous linear functional $V_{c}^{\infty}(X) \rightarrow \mathbb{C}$ called the integration functional.

\subsection{Valuations with compact support.}

In this subsection we introduce the space of valuations $V_{c}^{\infty}(X)$ with compact support and establish some of the simplest properties of it.

Let $\phi \in V^{\infty}(X)$. We say that a point $x \in X$ does not belong to the support of $\phi$ if there exists a neighborhood $U$ of $x$ such that $\left.\phi\right|_{U} \equiv 0$. The set of all points which does not belong to support of $\phi$ is an open subset of $X$. Its complement is called the support of $\phi$ and is denoted by supp $\phi$. Thus supp $\phi$ is a closed subset of $X$. The following lemma is obvious.

5.1.1 Lemma. For any $\phi, \psi \in V^{\infty}(X)$

$$
\operatorname{supp}(\phi \cdot \psi) \subset \operatorname{supp} \phi \cap \operatorname{supp} \psi \text {. }
$$

The space of all valuations with compact support will be denoted by $V_{c}^{\infty}(X)$. Also for any subset $S \subset X$ let us denote

$$
V_{S}^{\infty}(X):=\left\{\phi \in V^{\infty}(X) \mid \operatorname{supp} \phi \subset S\right\} .
$$

By Lemma 5.1.1 $V_{S}^{\infty}(X)$ is a subalgebra of $V^{\infty}(X)$ (without unit, unless $S=X$ ). If $S$ is closed then $V_{S}^{\infty}(X)$ is a closed subalgebra in $V^{\infty}(X)$. Also

$$
V_{c}^{\infty}(X)=\cup_{S \text { compact }} V_{S}^{\infty}(X)=\underset{S \text { compact }}{\lim _{S}} V_{S}^{\infty}(X) .
$$

Let us equip $V_{c}^{\infty}(X)=\underset{S \text { compact }}{\lim } V_{S}^{\infty}(X)$ with the linear topology of inductive limit when each space $V_{S}^{\infty}(X)$ is equipped with the topology induced from $V^{\infty}(X)$. It is easy to see that $V_{c}^{\infty}(X)$ is a locally convex Hausdorff linear topological space. The identical imbedding $V_{c}^{\infty}(X) \hookrightarrow V^{\infty}(X)$ is continuous.

For any subset $S \subset X$ let us denote

$$
\begin{aligned}
W_{i, S} & :=V_{S}^{\infty}(X) \cap W_{i}(X), \\
W_{i, c} & :=V_{c}^{\infty}(X) \cap W_{i}(X) .
\end{aligned}
$$

If $S$ is closed then $W_{i, S} \subset W_{i}(X)$ is a closed subspace. We will need the following lemma.

5.1.2 Lemma. Let $S$ be a closed subset of $X$. Then for any $j=0, \ldots, n$

$$
H_{S}^{i}\left(X, \mathcal{W}_{j}\right)=0 \text { for } i>0
$$

where $H_{S}^{i}$ denotes the $i$-th cohomology with support in $S$. 
Proof. The sheaf $\mathcal{W}_{j}$ has the descending filtration

$$
\mathcal{W}_{j} \supset \mathcal{W}_{j+1} \supset \cdots \supset \mathcal{W}_{n}
$$

It is enough to show that for any $p H_{S}^{i}\left(X, \mathcal{W}_{p} / \mathcal{W}_{p+1}\right)=0$ for $i>0$. Let us denote by $\mathcal{O}_{X}$ the sheaf of $C^{\infty}$-smooth functions on $X$. Then $\mathcal{W}_{p} / \mathcal{W}_{p+1}$ is a sheaf of $\mathcal{O}_{X}$-modules. It is well known (see e.g. [13], Section 3.7 of Ch. II) that on any smooth manifold $X$, for any sheaf $\mathcal{F}$ of $\mathcal{O}_{X}$-modules, and any closed subset $S \subset X$ one has

$$
H_{S}^{i}(X, \mathcal{F})=0 \text { for } i>0 .
$$

This implies the lemma. Q.E.D.

5.1.3 Lemma. (1) For any closed subset $S \subset X$ the canonical isomorphism

$$
W_{i} / W_{i+1} \tilde{\rightarrow} C^{\infty}\left(X, \operatorname{Val}_{i}^{s m}(T X)\right)
$$

induces isomorphism

$$
W_{i, S} / W_{i+1, S} \tilde{\rightarrow} C_{S}^{\infty}\left(X, \operatorname{Val}_{i}^{s m}(T X)\right)
$$

where $C_{S}^{\infty}$ stays for the space of infinitely smooth sections with support in $S$.

(2) Similarly the isomorphism (5.1.2) indices isomorphism

$$
W_{i, c} / W_{i+1, c} \stackrel{\sim}{\rightarrow} C_{c}^{\infty}\left(X, \operatorname{Val}_{i}^{s m}(T X)\right) .
$$

Proof. Part (2) follows from part (1) by passing to direct limit. Thus let us prove part (1). Equality (3.1.2) implies that we have a short exact sequence of sheaves on $X$

$$
0 \rightarrow \mathcal{W}_{i+1} \rightarrow \mathcal{W}_{i} \rightarrow \underline{\operatorname{Val}}_{i}(T X) \rightarrow 0 .
$$

Taking sections with the support in $S$ we obtain the following exact sequence

$$
0 \rightarrow W_{i+1, S} \rightarrow W_{i, S} \rightarrow C_{S}^{\infty}\left(X, \operatorname{Val}_{i}^{s m}(T X)\right) \rightarrow H_{S}^{1}\left(X, \mathcal{W}_{i+1}\right) .
$$

But by Lemma $5.1 .2 H_{S}^{1}\left(X, \mathcal{W}_{i+1}\right)=0$. Hence the result follows. Q.E.D.

\subsection{The integration functional.}

In this subsection we are going to introduce a canonical linear functional

$$
\int: V_{c}^{\infty}(X) \rightarrow \mathbb{C}
$$

which we call the integration functional. With slight oversimplification

$$
\int \phi=\phi(X)
$$

for any $\phi \in V_{c}^{\infty}(X)$. This definition is formally correct if $X$ is compact. Otherwise $X \notin$ $\mathcal{P}(X)$, and the above definition requires an explanation.

Let us construct the integration functional formally for general manifold $X$. First fix a compact subset $S \subset X$. Let us choose a compact subset $S^{\prime}$ with smooth boundary and such that $S$ in contained in the interior of $S^{\prime}$. Then $S^{\prime} \in \mathcal{P}(X)$. For any $\phi \in V_{S}^{\infty}(X)$ define

$$
S \int \phi:=\phi\left(S^{\prime}\right) \text {. }
$$


5.2.1 Lemma. (1) ${ }^{S} \int: V_{S}^{\infty}(X) \rightarrow \mathbb{C}$ is a continuous linear functional.

(2) For fixed $S$, the right hand side in (5.2.1) is independent of $S^{\prime}$ containing $S$.

Proof. Part (1) is obvious. Let us prove part (2). Let $S^{\prime \prime}$ be another compact subset with smooth boundary containing $S$ in the interior. We have to show that $\phi\left(S^{\prime}\right)=\phi\left(S^{\prime \prime}\right)$. Choosing a larger subset if necessary one may assume that $S^{\prime}$ is contained in the interior of $S^{\prime \prime}$. Then

$$
\phi\left(S^{\prime \prime}\right)=\phi\left(S^{\prime}\right)+\phi\left(\overline{S^{\prime \prime} \backslash S^{\prime}}\right)-\phi\left(\partial S^{\prime}\right)=\phi\left(S^{\prime}\right)
$$

where the last equality is due to the fact that supp $\phi \subset S \subset$ int $S^{\prime}$. Q.E.D.

As in the proof of Lemma 5.2.1 it is easy to see that if $S_{1} \subset S_{2}$ then the restriction of $S_{2} \int$ to $V_{S_{1}}^{\infty}(X)$ is equal to ${ }^{S_{1}} \int$. Thus we obtain a continuous linear functional

$$
\int: V_{c}^{\infty}(X) \rightarrow \mathbb{C}
$$

5.2.2 Remark. The space of smooth compactly supported densities is a subspace of $V_{c}^{\infty}(X)$; it is equal to $W_{n, c}$. The restriction of the above constructed integration functional $\int$ to this subspace coincides with the usual integration of densities.

\section{The selfduality property of valuations.}

The goal of this section is to establish the selfduality property of valuations (Theorem 6.1.1. Subsection 6.1). Subsection 6.2 contains a technical result on partition of unity in valuations.

\subsection{The selfduality property.}

Probably the most interesting property of the multiplicative structure on valuations is Theorem 6.1.1 below. Its prove heavily uses the Irreducibility Theorem for translation invariant valuations from $[2]$.

6.1.1 Theorem. Consider the bilinear form

$$
V^{\infty}(X) \times V_{c}^{\infty}(X) \rightarrow \mathbb{C}
$$

given by $(\phi, \psi) \mapsto \int \phi \cdot \psi$.

This bilinear form is a perfect pairing. More precisely the induced map

$$
V^{\infty}(X) \rightarrow\left(V_{c}^{\infty}(X)\right)^{*}
$$

is injective and has a dense image with respect to the weak topology on $\left(V_{c}^{\infty}(X)\right)^{*}$.

Theorem 6.1.1 follows from the next more precise statement by application of the HahnBanach theorem.

6.1.2 Theorem. (1) For any $\phi \in W_{i} \backslash W_{i+1}$ there exists $\psi \in W_{n-i, c}$ such that $\int \phi \cdot \psi \neq 0$.

(2) Similarly for any $\phi \in W_{i, c} \backslash W_{i+1, c}$ there exists $\psi \in W_{n-i}$ such that $\int \phi \cdot \psi \neq 0$. 
Proof. The proves of these two statements are very similar. Thus let us prove only the first one. Let $\phi \in W_{i} \backslash W_{i+1}$. Let us denote by $\tilde{\phi}$ the image of $\phi$ is $W_{i} / W_{i+1}=$ $C^{\infty}\left(X, \operatorname{Val}_{i}^{s m}(T X)\right)$. Thus $\tilde{\phi} \neq 0$. We will show that there exists $\psi \in W_{n-i, c}$ such that $\int \phi \cdot \psi \neq 0$. Since $W_{i+1} \cdot W_{n-i}=0$ and $W_{i} \cdot W_{n-i+1}=0$, the product $\phi \cdot \psi$ depends only on $\tilde{\phi}$ and on the image $\tilde{\psi}$ of $\psi$ in $W_{n-i, c} / W_{n-i+1, c}=C_{c}^{\infty}\left(X, V a l_{n-i}^{s m}(T X)\right)$ (where the last equality is due to Lemma 5.1.3. $(2)$.

Thus it is enough to show that for any $\tilde{\phi} \in C^{\infty}\left(X, \operatorname{Val}_{i}^{s m}(T X)\right)$ there exists $\tilde{\psi} \in$ $C_{c}^{\infty}\left(X, \operatorname{Val}_{n-i}^{s m}(T X)\right)$ such that

$$
\int_{X} \tilde{\phi} \cdot \tilde{\psi} \neq 0
$$

where the product $\tilde{\phi} \cdot \tilde{\psi}$ is understood pointwise in the tangent space of each point, $\tilde{\phi} \cdot \tilde{\psi} \in$ $C_{c}^{\infty}\left(X, \operatorname{Val}_{n}^{s m}(T X)\right)=C_{c}^{\infty}\left(X,\left|\omega_{X}\right|\right)$, and the integration is understood in the sense of the usual integration of densities.

Let us fix a point $x_{0} \in X$ such that $\tilde{\phi}\left(x_{0}\right) \neq 0$. By the Poincaré duality for the translation invariant valuations (Theorem 0.8 in [3] ) there exists $\xi_{0} \in \operatorname{Val}_{n-i}^{s m}\left(T_{x_{0}} X\right)$ such that $\tilde{\phi}\left(x_{0}\right) \cdot \xi_{0} \neq$ 0 . Let $\xi \in C^{\infty}\left(X, \operatorname{Val}_{n-i}^{s m}(T X)\right)$ be a section such that $\xi\left(x_{0}\right)=\xi_{0}$.

Consider the following $C^{\infty}$-smooth density on $X$

$$
\tau:=\tilde{\phi} \cdot \xi .
$$

Thus $\tau\left(x_{0}\right) \neq 0$. Hence we can find a smooth compactly supported function $\delta \in C_{c}^{\infty}(X)$ such that $\int_{X} \tau \cdot \delta \neq 0$. Take $\tilde{\psi}:=\xi \cdot \delta$. Then

$$
\int \tilde{\phi} \cdot \tilde{\psi}=\int_{X} \tau \cdot \delta \neq 0
$$

Q.E.D.

From Theorem 6.1.2 we immediately deduce the following corollary.

\subsubsection{Corollary.}

$$
\begin{aligned}
& W_{i}=\left\{\phi \in V^{\infty}(X) \mid \int \phi \cdot \psi=0 \text { for any } \psi \in W_{n-i+1, c}\right\}, \\
& W_{i, c}=\left\{\phi \in V_{c}^{\infty}(X) \mid \int \phi \cdot \psi=0 \text { for any } \psi \in W_{n-i+1}\right\} .
\end{aligned}
$$

\subsection{Partition of unity in valuations.}

6.2.1 Proposition. Let $\left\{U_{\alpha}\right\}_{\alpha}$ be a locally finite open covering of a manifold $X$. Then there exist $\left\{\phi_{\alpha}\right\}_{\alpha} \subset V^{\infty}(X)$ such that

$$
\operatorname{supp}\left(\phi_{\alpha}\right) \subset U_{\alpha} \text { and } \sum_{\alpha} \phi_{\alpha} \equiv \chi
$$

where the sum is locally finite, and $\chi$ denotes the Euler characteristic.

Proposition 6.2.1 is an immediate consequence of the fact that the sheaf $\mathcal{V}_{X}^{\infty}$ of smooth valuations is soft (by Proposition 3.1.8 of [5] ) and the following general result. 
6.2.2 Proposition ([13], Theorem 3.6.1, Ch. II). Let $X$ be a paracompact topological space. Let $\left\{U_{i}\right\}_{i \in I}$ be a locally finite open covering of $X$. Let $\mathcal{L}$ be a soft sheaf over $X$. Then for any section $s \in \mathcal{L}(X)$ there exists a collection of sections $\left\{s_{i}\right\}_{i \in I} \subset \mathcal{L}(X)$ such that

(1) $\operatorname{supp} s_{i} \subset U_{i}$;

(2) the family of subsets $\left\{\operatorname{supp} s_{i}\right\}_{i \in I}$ is locally finite;

(3) $s=\sum_{i \in I} s_{i}$.

\section{Generalized valuations.}

In this section we introduce and study the space $V^{-\infty}(X)$ of generalized valuations. It is defined in Subsection [7.1. In Subsection [7.2] it is shown that generalized valuations form naturally a sheaf on $X$; it is a sheaf of modules over the sheaf of algebras of smooth valuations. In Subsection 7.3 a canonical filtration on generalized valuations is introduced and studied; it extends in a sense the canonical filtration on smooth valuations. In Subsection 7.4 we extend the Euler-Verdier involution from smooth valuations to generalized ones.

\subsection{The space of generalized valuations.}

7.1.1 Definition. Define the space of generalized valuations by

$$
V^{-\infty}(X):=\left(V_{c}^{\infty}(X)\right)^{*}
$$

equipped with the usual weak topology on the dual space.

7.1.2 Remark. It is important to observe that by Theorem 6.1.1 we have a canonical imbedding

$$
V^{\infty}(X) \hookrightarrow V^{-\infty}(X)
$$

with the image dense in the weak topology. Thus we can consider the space of generalized valuations as a completion of the space of smooth compactly supported valuations with respect to the weak topology.

Let us describe on $V^{-\infty}(X)$ the canonical structure of $V^{\infty}(X)$-module. Let $\xi \in V^{\infty}(X), \psi \in$ $V^{-\infty}(X)$. Define their product $\xi \cdot \psi$ by

$$
<\xi \cdot \psi, \phi>=<\phi, \xi \cdot \phi>
$$

for any $\phi \in V_{c}^{\infty}(X)$. Clearly this defines a map

$$
\mu: V^{\infty}(X) \times V^{-\infty}(X) \rightarrow V^{-\infty}(X) .
$$

7.1.3 Proposition. The map $\mu$ is a separately continuous bilinear map. It defines a structure of $V^{\infty}(X)$-module on $V^{-\infty}(X)$. Moreover $V^{\infty}(X)$ is a submodule of $V^{-\infty}(X)$, and the induced structure of $V^{\infty}(X)$-module on it is the standard one. 
Proof. The bilinearity is obvious from the definition. Let us check the continuity. We have to check that for any $\phi \in V_{c}^{\infty}(X)$ the map

$$
V^{\infty}(X) \times V^{-\infty}(X) \rightarrow \mathbb{C}
$$

given by $(\xi, \psi) \mapsto\langle\psi, \xi \cdot \phi\rangle$ is separately continuous. But this is an immediate consequence of the continuity of the map $V^{\infty}(X) \rightarrow V_{c}^{\infty}(X)$ given by $\xi \mapsto \xi \cdot \phi$ and separate continuity of the canonical pairing $V_{c}^{\infty}(X) \times V^{-\infty}(X) \rightarrow \mathbb{C}$.

Let us check now that the above map $\mu: V^{\infty}(X) \times V^{-\infty}(X) \rightarrow V^{-\infty}(X)$ defines the standard $V^{\infty}(X)$-module structure on $V^{\infty}(X) \hookrightarrow V^{-\infty}(X)$. Namely we have to show that for $\xi, \psi \in V^{\infty}(X)$ one has $\mu(\xi, \psi)=\xi \cdot \psi$ where the last product is understood in the usual sense. Let $\phi \in V_{c}^{\infty}(X)$. Then we have

$$
\begin{array}{r}
<\mu(\xi, \psi), \phi>=<\psi, \xi \cdot \phi>= \\
\int \psi \cdot(\xi \cdot \phi)=\int(\xi \cdot \psi) \cdot \phi=<\xi \cdot \psi, \phi>.
\end{array}
$$

Hence $\mu(\xi, \phi)=\xi \cdot \psi$.

Since $V^{\infty}(X)$ is dense in $V^{-\infty}(X)$ and $\mu$ is continuous it follows that $\mu$ defines $V^{\infty}(X)$ module structure on $V^{-\infty}(X)$. Q.E.D.

\subsection{The sheaf property of generalized valuations.}

In this subsection we describe the canonical sheaf structure on generalized valuations.

First observe that for two open subsets $U_{1} \subset U_{2}$ of a manifold $X$ we have the identity imbedding

$$
V_{c}^{\infty}\left(U_{1}\right) \hookrightarrow V_{c}^{\infty}\left(U_{2}\right)
$$

Hence by duality we have a continuous map

$$
V^{-\infty}\left(U_{2}\right) \rightarrow V^{-\infty}\left(U_{1}\right)
$$

7.2.1 Lemma. The map (7.2.2) being restricted to $V^{\infty}\left(U_{2}\right) \subset V^{-\infty}\left(U_{2}\right)$ coincides with the usual restriction map $V^{\infty}\left(U_{2}\right) \rightarrow V^{\infty}\left(U_{1}\right)$.

Proof. Let us denote temporarily the imbedding (7.2.1) by $\tau$, and its dual (7.2.2) by $\tau^{*}$. Let $\phi \in V^{\infty}\left(U_{2}\right)$. Then for any $\psi \in V_{c}^{\infty}\left(U_{1}\right)$ one has

$$
<\tau^{*}(\phi), \psi>=(\phi \cdot \tau(\psi))\left(U_{2}\right)=\left(\left.\phi\right|_{U_{1}} \cdot \psi\right)\left(U_{1}\right)=<\left.\phi\right|_{U_{1}}, \psi>.
$$

Hence $\tau^{*}(\phi)=\left.\phi\right|_{U_{1}}$. Q.E.D.

7.2.2 Proposition. The assignment

$$
U \mapsto V^{-\infty}(U)
$$

to any open subset $U \subset X$ with the above restriction maps defines a sheaf on $X$ denoted by $\mathcal{V}_{X}^{-\infty}$ 
7.2.3 Remark. Given this proposition, it is clear that $\mathcal{V}_{X}^{\infty}$ is a subsheaf of $\mathcal{V}_{X}^{-\infty}$.

Proof of Proposition 7.2.2 Let $\left\{U_{\alpha}\right\}_{\alpha}$ be an open covering of an open subset $U$. Let $\phi \in V^{-\infty}(V)$ such that $\left.\phi\right|_{U_{\alpha}}=0$ for any $\alpha$. Let us show that $\phi=0$. Replacing $\left\{U_{\alpha}\right\}$ by a refinement we may assume that $\left\{U_{\alpha}\right\}$ is locally finite. Let us choose a partition of unity $\left\{\phi_{\alpha}\right\}$ subordinate to this covering using Proposition 6.2.1. For any $\psi \in V_{c}^{\infty}(X)$ we have

$$
\begin{array}{r}
<\phi, \psi>=<\phi, \sum_{\alpha} \phi_{\alpha} \cdot \psi>= \\
\sum_{\alpha}<\phi, \phi_{\alpha} \cdot \psi>=\sum_{\alpha}<\left.\phi\right|_{U_{\alpha}},\left(\phi_{\alpha} \cdot \psi\right)_{U_{\alpha}}>=0 .
\end{array}
$$

Hence $\phi=0$.

Now let us assume that we are given an open covering $\left\{U_{\alpha}\right\}_{\alpha}$ of an open subset $U \subset X$, and for any $\alpha$ we are given a generalized valuation $\psi_{\alpha} \in V^{-\infty}\left(U_{\alpha}\right)$ such that $\left.\psi_{\alpha}\right|_{U_{\alpha} \cap U_{\beta}}=$ $\left.\psi_{\beta}\right|_{U_{\alpha} \cap U_{\beta}}$ for any $\alpha, \beta$. Let us show that there exists $\psi \in V^{-\infty}(U)$ such that $\left.\psi\right|_{U_{\alpha}}=\psi_{\alpha}$. Again by choosing a refinement we may assume that the covering $\left\{U_{\alpha}\right\}$ is locally finite. Let us fix a partition of unity $\left\{\phi_{\alpha}\right\}$ subordinate to it. Define $\psi$ by

$$
<\psi, \phi>:=\sum_{\alpha}<\psi_{\alpha},\left.\left(\phi_{\alpha} \cdot \phi\right)\right|_{U_{\alpha}}>
$$

for any $\phi \in V_{c}^{\infty}(U)$. It is easy to see that $\psi \in V^{-\infty}(U)$ and $\left.\psi\right|_{U_{\alpha}}=\psi_{\alpha}$. Q.E.D.

7.2.4 Proposition. Being equipped with the above restriction maps and the defined above product of generalized valuations by smooth ones, $\mathcal{V}_{X}^{-\infty}$ is a sheaf of $\mathcal{V}_{X}^{\infty}$-modules.

Proof. For an open subset $U \subset X$ let us denote by

$$
\mu_{U}: V^{\infty}(U) \times V^{-\infty}(U) \rightarrow V^{-\infty}(U)
$$

the canonical product. We have to check that for any open subsets $U \subset V \subset X$, any $\xi \in V^{\infty}(V), \psi \in V^{-\infty}(V)$ one has

$$
\left.\left(\mu_{V}(\xi, \psi)\right)\right|_{U}=\mu_{U}\left(\left.\xi\right|_{U},\left.\psi\right|_{U}\right) .
$$

Let $\phi \in V_{c}^{\infty}(U)$. Let us denote the identity imbedding $V_{c}^{\infty}(U) \hookrightarrow V_{c}^{\infty}(V)$ by $\tau$.

Then we have

$$
\begin{gathered}
<\left.\left(\mu_{V}(\xi, \psi)\right)\right|_{U}, \phi>=<\mu_{V}(\xi, \psi), \tau(\phi)>=<\psi, \xi \cdot \tau(\phi)>= \\
<\psi, \tau\left(\left.\xi\right|_{U} \cdot \phi\right)>=<\left.\psi\right|_{U},\left.\xi\right|_{U} \cdot \phi>=<\mu_{U}\left(\left.\xi\right|_{U},\left.\psi\right|_{U}\right), \phi>.
\end{gathered}
$$

Hence (7.2.3) follows. Q.E.D.

\subsection{Filtration on generalized valuations.}

7.3.1 Definition. Define $W_{i}\left(V^{-\infty}(X)\right)$ to be the closure of $W_{i}(X)\left(\subset V^{\infty}(X) \subset V^{-\infty}(X)\right)$ in the space $V^{-\infty}(X)$ with respect to the weak topology. 
Clearly one has

$$
V^{-\infty}(X)=W_{0}\left(V^{-\infty}(X)\right) \supset W_{1}\left(V^{-\infty}(X)\right) \supset \cdots \supset W_{n}\left(V^{-\infty}(X)\right) .
$$

In this subsection we will also use the following notation. The subspace $W_{i}(X)$ of $V^{\infty}(X)$ will also be denoted by $W_{i}\left(V^{\infty}(X)\right)$. Set

$$
\begin{array}{r}
W_{i}\left(V_{c}^{\infty}(X)\right):=W_{i}\left(V^{\infty}(X)\right) \cap V_{c}^{\infty}(X), \\
W_{i}\left(V_{c}^{-\infty}(X)\right):=W_{i}\left(V^{-\infty}(X)\right) \cap V_{c}^{-\infty}(X) .
\end{array}
$$

It is easy to see (using the separate continuity of the product $V^{\infty}(X) \times V^{-\infty}(X) \rightarrow V^{-\infty}(X)$ ) that

$$
W_{i}\left(V^{\infty}(X)\right) \cdot W_{j}\left(V^{-\infty}(X)\right) \subset W_{i+j}\left(V^{-\infty}(X)\right)
$$

7.3.2 Proposition. For any $i=0,1, \ldots, n$

$$
W_{i}\left(V^{-\infty}(X)\right)=\left\{\phi \in V^{-\infty}(X) \mid<\phi, \psi>=0 \text { for any } \psi \in W_{n-i+1}\left(V_{c}^{\infty}(X)\right)\right\} .
$$

Proof. Let us denote by $W_{i}^{\prime}\left(V^{-\infty}(X)\right)$ the space in the right hand side of (7.3.2). The equality (7.3.1) implies that

$$
W_{i}\left(V^{-\infty}(X)\right) \subset W_{i}^{\prime}\left(V^{-\infty}(X)\right)
$$

Let us prove the converse inclusion. Let us assume in the contrary that there exists $\psi \in$ $W_{i}^{\prime}\left(V^{-\infty}(X)\right) \backslash W_{i}\left(V^{-\infty}(X)\right)$. Since $W_{i}\left(V^{-\infty}(X)\right)$ is a closed subspace of $V^{-\infty}(X)$ in the weak topology, the Hahn-Banach theorem implies that there exists $\phi \in V_{c}^{\infty}(X)$ such that $<\psi, \phi>\neq 0$ and for any $\xi \in W_{i}\left(V^{-\infty}(X)\right)$

$$
<\xi, \phi>=0 \text {. }
$$

Since $W_{i}\left(V^{\infty}(X)\right) \subset W_{i}\left(V^{-\infty}(X)\right)$ Corollary 6.1.3 implies that $\phi \in W_{n-i+1}\left(V_{c}^{\infty}(X)\right)$. But then (7.3.1) implies that $\langle\psi, \phi\rangle=0$. This is a contradiction. Q.E.D.

\subsubsection{Corollary.}

$$
W_{i}\left(V^{-\infty}(X)\right) \cap V^{\infty}(X)=W_{i}\left(V^{\infty}(X)\right) .
$$

Proof. This immediately follows from Proposition [7.3.2 and Corollary 6.1.3, Q.E.D.

For a subset $S \subset X$ let us denote by $V_{S}^{-\infty}(X)$ the space of generalized valuations with support contained in $S$. Clearly $V_{S}^{-\infty}(X)$ is a $V^{\infty}(X)$-submodule of $V^{-\infty}(X)$. If $S$ is a closed subset of $X$ then $V_{S}^{-\infty}(X)$ is a closed subspace of $V^{-\infty}(X)$ in the weak topology. It is easy to see that

$$
V_{c}^{-\infty}(X)=\lim _{S \text { compact }} V_{S}^{-\infty}(X) .
$$

Let us equip $V_{c}^{-\infty}(X)$ with the topology of inductive limit when each of $V_{S}^{-\infty}(X)$ is equipped with the topology induced from $V^{-\infty}(X)$. Then $V_{c}^{-\infty}(X)$ is a locally convex Hausdorff linear topological space. 
7.3.4 Proposition. For any $i=0,1, \ldots, n$, the space $W_{i}\left(V_{c}^{\infty}(X)\right)$ is dense in $W_{i}\left(V_{c}^{-\infty}(X)\right)$ in the above topology of inductive limit.

Proof. Fix $\phi \in W_{i}\left(V_{c}^{-\infty}(X)\right)$. Set $S:=\operatorname{supp} \phi$ be the support of $\phi . S$ is a compact set. Let $U$ be an open relatively compact neighborhood of $S$. Since the sheaf $\mathcal{V}_{X}^{\infty}$ of smooth valuations is soft (by Proposition 3.1.8 of [5] ), there exists $\alpha \in V^{\infty}(X)$ such that $\alpha$ is equal to the Euler characteristic $\chi$ in a neighborhood of $S$, and $\left.\alpha\right|_{X \backslash U} \equiv 0$.

Since $W_{i}\left(V^{\infty}(X)\right)$ is dense in $W_{i}\left(V^{-\infty}(X)\right)$ in the weak topology, there exists a net $\left\{\phi_{\lambda}\right\} \subset$ $W_{i}\left(V^{\infty}(X)\right)$ which converges to $\phi$ in the weak topology. But then $\left\{\alpha \cdot \phi_{\lambda}\right\} \subset W_{i}\left(V_{U}^{\infty}(X)\right)$, and $\left\{\alpha \cdot \phi_{\lambda}\right\}$ converges to $\alpha \cdot \phi=\phi$ in $V_{c}^{-\infty}(X)$. Q.E.D.

7.3.5 Proposition. Let $n=\operatorname{dim} X$ as previously. Then there exists a canonical isomorphism of linear topological spaces

$$
W_{n}\left(V^{-\infty}(X)\right)=C^{-\infty}\left(X,\left|\omega_{X}\right|\right) .
$$

Proof. By Proposition 7.3 .2 one has

$$
\begin{aligned}
W_{n}\left(V^{-\infty}(X)\right)=W_{1}\left(V_{c}^{\infty}(X)\right)^{\perp}= & \left(V_{c}^{\infty}(X) / W_{1}\left(V_{c}^{\infty}(X)\right)\right)^{*}= \\
& \left(C_{c}^{\infty}(X)\right)^{*}=C^{-\infty}\left(X,\left|\omega_{X}\right|\right)
\end{aligned}
$$

where the third equality is due to Lemma 5.1.3(2). Q.E.D.

7.3.6 Proposition. There exists a canonical isomorphism of linear topological spaces

$$
V^{-\infty}(X) / W_{1}\left(V^{-\infty}(X)\right)=C^{-\infty}(X) .
$$

Proof. Using Proposition 7.3.2 one has

$$
\begin{array}{r}
V^{-\infty}(X) / W_{1}\left(V^{-\infty}(X)\right)=V_{c}^{\infty}(X)^{*} / W_{n}\left(V_{c}^{\infty}(X)\right)^{\perp}= \\
W_{n}\left(V_{c}^{\infty}(X)\right)^{*}=\left(C_{c}^{\infty}\left(X,\left|\omega_{X}\right|\right)\right)^{*}=C^{-\infty}(X) .
\end{array}
$$

Q.E.D.

Remind that by Proposition 7.3.4 $V_{c}^{\infty}(X)$ is dense in $V_{c}^{-\infty}(X)$ (in the topology of inductive limit).

7.3.7 Proposition. The integration functional

$$
\int: V_{c}^{\infty}(X) \rightarrow \mathbb{C}
$$

extends uniquely by continuity to the functional

$$
\int: V_{c}^{-\infty}(X) \rightarrow \mathbb{C} .
$$


Proof. First observe that for any $\alpha \in V_{c}^{\infty}(X)$ the functional $V^{\infty}(X) \rightarrow \mathbb{C}$ given by $\phi \mapsto \int \alpha \cdot \phi$ extends (uniquely) by continuity in the weak topology to $V^{-\infty}(X)$. Indeed this extension is given by $\psi \mapsto<\psi, \alpha>$. Let us denote this functional by $\hat{\alpha}$. Thus $\hat{\alpha}: V^{-\infty}(X) \rightarrow$ $\mathbb{C}$ is a continuous functional.

Let us fix an arbitrary compact subset $S \subset X$. Let us fix a smooth compactly supported valuation $\alpha \in V_{c}^{\infty}(X)$ such that $\alpha$ equals to the Euler characteristic in a neighborhood of $S$. Consider the corresponding continuous linear functional $\hat{\alpha}: V^{-\infty}(X) \rightarrow \mathbb{C}$. We claim that the restriction of $\hat{\alpha}$ to $V_{S}^{-\infty}(X)$ is the desired extension of the integration functional to $V_{S}^{-\infty}(X)$.

To check it let us fix a compact neighborhood $S^{\prime}$ of $S$ such that the restriction of $\alpha$ to $S^{\prime}$ is still equal to the Euler characteristic. By (the proof of) Proposition 7.3.4 every valuation from $V_{S}^{-\infty}(X)$ can be approximated in the weak topology by a net from $V_{S^{\prime}}^{\infty}(X)$. Hence it is enough to check that for any $\phi \in V_{S^{\prime}}^{\infty}(X)$ one has

$$
\int \alpha \cdot \phi=\int \phi
$$

But this is obvious since

$$
\phi \cdot(\alpha-\chi) \equiv 0
$$

Q.E.D.

7.3.8 Lemma. Let $\left\{\zeta_{\lambda}\right\}_{\lambda \in \Lambda} \subset V^{\infty}(X)$ be a net such that for any compact subset $K \subset X$ there exists $\lambda_{K} \in \Lambda$ such that for all $\lambda \geq \lambda_{K}$

$$
\left(\operatorname{supp} \zeta_{K}\right) \cap K=\emptyset \text {. }
$$

Then

$$
\lim _{\Lambda} \zeta_{\lambda}=0 \text { in } V^{\infty}(X)
$$

Proof. Consider the map

$$
T_{i}: \tilde{C}^{\infty}\left(T^{*} X, W_{i}\left(\Omega^{n} \otimes p^{*} o\right)\right) \rightarrow W_{i}\left(V^{\infty}(X)\right)
$$

given by the integration with respect to the characteristic cycle. By Proposition 3.1.3 of [5] $T_{i}$ is an epimorphism. By the definition of the topology on $V^{\infty}(X)$ (see Subsection 3.2 of 5] $T_{i}$ is a continuous map. Hence it is enough to show that for any compact subset $K \subset X$ there exists $\lambda_{K} \in \Lambda$ such that for any $\lambda \geq \lambda_{K}$ there exists $\eta_{\lambda} \in \tilde{C}^{\infty}\left(T^{*} X, \Omega^{n} \otimes p^{*} o\right)$ satisfying

(i) $T_{i}\left(\eta_{\lambda}\right)=\zeta_{\lambda}$;

(ii) $\eta_{\lambda}$ vanishes in a neighborhood of $p^{-1}(K)$.

Indeed then we would have

$$
\lim _{\Lambda} \zeta_{\lambda}=\lim _{\Lambda} T_{i}\left(\eta_{\lambda}\right)=0
$$

For the rest of the proof of the lemma let fix a compact subset $K \subset X$. As in Section 2 consider the sheaves on $X$

$$
\begin{array}{r}
\mathcal{W}_{i}(U)=W_{i}\left(V^{\infty}(U)\right), \\
\mathcal{W}_{i}^{\prime}(U)=\tilde{C}^{\infty}\left(T^{*} U, W_{i}\left(\Omega^{n} \otimes p^{*} o\right)\right)
\end{array}
$$


for any open subset $U \subset X$. The integration over the the characteristic cycle gives a morphism of sheaves

$$
T_{i}: \mathcal{W}_{i}^{\prime} \rightarrow \mathcal{W}_{i}
$$

which is an epimorphism (we denote this morphism by the same symbol $T_{i}$ ). Set again $\mathcal{K}_{i}:=\operatorname{Ker} T_{i}$. It was shown in [5] (see the proof of Proposition 3.1.9) that the sheaves $\mathcal{K}_{j} / \mathcal{K}_{j+1}$ are isomorphic to the sheaves of $\mathcal{O}_{X}$-modules where $\mathcal{O}_{X}$ denotes the sheaf of $C^{\infty}$ smooth functions on $X$. By Section 3.7 of Ch. II of [13] the sheaves $\mathcal{K}_{j} / \mathcal{K}_{j+1}$ are soft for any $j$. Hence for any closed subset $Z \subset X$ the positive cohomology groups with support in $Z$ vanish:

$$
H_{Z}^{i}\left(X, \mathcal{K}_{j} / \mathcal{K}_{j+1}\right)=0 \text { for } i>0 .
$$

Using the long exact sequence we get

$$
H_{Z}^{i}\left(X, \mathcal{K}_{j}\right)=0 \text { for } i>0 \text { and any } j .
$$

Consider the short exact sequence of sheaves

$$
0 \rightarrow \mathcal{K}_{i} \rightarrow \mathcal{W}_{i}^{\prime} \rightarrow \mathcal{W}_{i} \rightarrow 0 .
$$

From the long exact sequence we obtain

$$
H_{Z}^{0}\left(X, \mathcal{W}_{i}^{\prime}\right) \rightarrow H_{Z}^{0}\left(X, \mathcal{W}_{i}\right) \rightarrow H_{Z}^{1}\left(X, \mathcal{K}_{i}\right)=0
$$

for any closed subset $Z \subset X$. Namely the map

$$
H_{Z}^{0}\left(X, \mathcal{W}_{i}^{\prime}\right) \rightarrow H_{Z}^{0}\left(X, \mathcal{W}_{i}\right)
$$

is surjective.

Let us choose $Z$ as follows. Let $U$ be an open relatively compact neighborhood of $K$. Set $Z:=X \backslash U$. There exists $\lambda_{0} \in \Lambda$ such that for any $\lambda \geq \lambda_{0}$ one has $\left(\operatorname{supp} \zeta_{\lambda}\right) \cap U=\emptyset$. Then clearly $\zeta_{\lambda} \in H_{Z}^{0}\left(X, \mathcal{W}_{i}\right)$ for $\lambda \geq \lambda_{0}$. The surjectivity of the map (7.3.4) implies the lemma. Q.E.D.

7.3.9 Lemma. (1) For any $i=0,1, \ldots, n$ the space $W_{i}\left(V_{c}^{\infty}(X)\right)$ is dense in $W_{i}\left(V^{\infty}(X)\right)$.

(2) For any $i=0,1, \ldots, n$ the space $W_{i}\left(V_{c}^{-\infty}(X)\right)$ is dense in $W_{i}\left(V^{-\infty}(X)\right)$.

Proof. Let us prove first part (1). For any compact subset $K \subset X$ let us choose a compactly supported valuation $\tau_{K} \in V_{c}^{\infty}(X)$ such $\tau_{K}$ is equal to the Euler characteristic $\chi$ in a neighborhood of $K$. Let $\psi \in W_{i}\left(V^{\infty}(X)\right)$. It is enough to show that

$$
\lim _{K \text { compact }}\left(\tau_{K} \cdot \psi\right)=\psi \text { in } V^{\infty}(X) .
$$

Let us denote $\zeta_{K}:=\left(\tau_{K}-\chi\right) \cdot \psi$. Clearly $\zeta_{K}$ vanishes in a neighborhood of $K$. By Lemma 7.3.8 $\lim _{K \text { compact }} \zeta_{K}=0$.

Let us prove part (2). Fix $\psi \in W_{i}\left(V^{-\infty}(X)\right)$. For any compact subset $K \subset X$ let us fix a compactly supported smooth valuation $\tau_{K} \in V_{c}^{\infty}(X)$ which is equal to the Euler 
characteristic $\chi$ in a neighborhood of $K$. Let $\psi_{K}:=\tau_{K} \cdot \psi$. Clearly $\psi_{K} \in W_{i}\left(V_{c}^{-\infty}(X)\right)$. It suffices to show that

$$
\lim _{K \text { compact }} \psi_{K}=\psi \text { in } V^{-\infty}(X) .
$$

Let $\phi \in V_{c}^{\infty}(X)$. We have to show that

$$
\lim _{K \text { compact }}<\psi_{K}, \phi>=<\psi, \phi>.
$$

We have

$$
\begin{array}{r}
\lim _{K \text { compact }}<\psi_{K}, \phi>=\lim _{K \text { compact }}<\psi, \tau_{K} \cdot \phi>= \\
<\psi, \lim _{\text {compact }}\left(\tau_{K} \cdot \phi\right)>=<\psi, \phi>.
\end{array}
$$

Part (2) is proved too. Q.E.D.

Let us observe now that the bilinear map $V_{c}^{-\infty}(X) \times V^{\infty}(X) \rightarrow \mathbb{C}$ given by

$$
(\psi, \phi) \mapsto \int \phi \cdot \psi
$$

is separately continuous. Hence it defines a continuous map

$$
\theta: V_{c}^{-\infty}(X) \rightarrow V^{\infty}(X)^{*}
$$

where $V^{\infty}(X)^{*}$ is equipped with the weak topology, and a continuous map

$$
\theta^{\prime}: V^{\infty}(X) \rightarrow V_{c}^{-\infty}(X)^{*}
$$

where $V_{c}^{-\infty}(X)^{*}$ is equipped with the weak topology.

7.3.10 Proposition. The maps $\theta$ and $\theta^{\prime}$ are isomorphisms of linear spaces.

Proof. First observe that if the manifold $X$ is compact then the result follows immediately from the definitions. Let us assume that $X$ is not compact.

First let us check that $\theta$ is injective. Assume that $\psi \in \operatorname{Ker} \theta$. Since $\psi \in V_{c}^{-\infty}(X) \subset$ $V^{-\infty}(X)=V_{c}^{\infty}(X)^{*}$, then for any $\phi \in V_{c}^{\infty}(X)$ one has $\langle\psi, \phi\rangle=0$. Hence $\psi=0$.

Let us check now that $\theta$ is onto. Let $\zeta \in V^{\infty}(X)^{*}$. Since the identity imbedding $V_{c}^{\infty}(X) \hookrightarrow V^{\infty}(X)$ is continuous, the restriction $\tilde{\zeta}$ of $\zeta$ to $V_{c}^{\infty}(X)$ is a continuous functional on $V_{c}^{\infty}(X)$, i.e. belongs to $V_{c}^{\infty}(X)^{*}=V^{-\infty}(X)$. Let us show that supp $\tilde{\zeta}$ is compact, i.e. $\tilde{\zeta} \in V_{c}^{-\infty}(X)$. Assume in the contrary that supp $\tilde{\zeta}$ is not compact. It means that for any compact subset $K \subset X$ there exists a valuation $\phi \in V_{c}^{\infty}(X)$ with supp $\phi \cap K=\emptyset$ such that $\langle\tilde{\zeta}, \phi>\neq 0$. Since we have assumed that $X$ is not compact we can construct an open covering $\left\{U_{\alpha}\right\}_{\alpha}$ of $X$ which does not have a finite subcovering. Since any manifold is paracompact (by definition) and locally compact, by choosing a refinement if necessary we may assume that this covering is locally finite and any $U_{\alpha}$ is relatively compact. Let us choose $U_{\alpha_{1}}$ so that $\operatorname{supp} \tilde{\zeta} \cap U_{\alpha_{1}} \neq \emptyset$. Denote $K_{1}:=\bar{U}_{\alpha_{1}}$. Assume we have constructed compact sets $K_{1}, \ldots, K_{N-1}$ with the following properties:

1) for each $i=1, \ldots, N-1$ there exists $\alpha_{i}$ such that $K_{i}=\bar{U}_{\alpha_{i}}$; 
2) the interior of $K_{i}$ intersects supp $\tilde{\zeta}$ non-trivially for each $i=1, \ldots, N-1$;

3) $K_{i} \cap K_{j}=\emptyset$ for $1 \leq i \neq j \leq N-1$.

Let us construct $K_{N}$ such that the sequence of sets $K_{1}, \ldots, K_{N-1}, K_{N}$ has the same properties. Let us fix an open relatively compact neighborhood $T$ of the set $\cup_{i=1}^{N-1} K_{i}$. Since the covering $\left\{U_{\alpha}\right\}$ is locally finite, and supp $\tilde{\zeta}$ is not compact, there exists $\alpha_{N}$ such that $U_{\alpha_{N}} \cap T=\emptyset$ and $U_{\alpha_{N}} \cap \operatorname{supp} \tilde{\zeta} \neq \emptyset$. Set $K_{N}:=\bar{U}_{\alpha_{N}}$. Then $K_{N} \cap\left(\cup_{i=1}^{N-1} K_{i}\right)=\emptyset$ and $K_{N} \cap \operatorname{supp} \tilde{\zeta} \neq \emptyset$. By induction we obtain an infinite sequence of pairwise disjoint compact sets $\left\{K_{N}\right\}_{N \in \mathbb{N}}$ with non-empty interiors such that int $K_{N} \cap \operatorname{supp} \tilde{\zeta} \neq \emptyset$ for any $N \in \mathbb{N}$.

Since $i n t K_{N} \cap \operatorname{supp} \tilde{\zeta} \neq \emptyset$ we can choose a valuation $\phi_{N} \in V^{\infty}(X)$ with supp $\phi_{N} \subset$ int $K_{N}$ and such that $<\tilde{\zeta}, \phi_{N}>=1$. Let us define

$$
\phi:=\sum_{N=1}^{\infty} \phi_{N}
$$

This series converges in $V^{\infty}(X)$ by Lemma 7.3.8, Then

$$
<\zeta, \phi>=\lim _{N \rightarrow \infty}<\zeta, \sum_{n=1}^{N} \phi_{n}>=\lim _{N \rightarrow \infty} N=\infty
$$

This is a contradiction. Hence we have shown that supp $\tilde{\zeta}$ is compact.

Let us show that $\zeta=\theta(\tilde{\zeta})$. For any $\phi \in V_{c}^{\infty}(X)$ we have

$$
<\zeta, \phi>=<\tilde{\zeta}, \phi>
$$

Hence $\left\langle\zeta, \phi>=<\theta(\tilde{\zeta}), \phi>\right.$ for any $\phi \in V_{c}^{\infty}(X)$. But by Lemma $7.3 .9 V_{c}^{\infty}(X)$ is dense in $V^{\infty}(X)$. Hence $\zeta=\theta(\tilde{\zeta})$. Thus we have shown that $\theta: V_{c}^{-\infty}(X) \rightarrow V^{\infty}(X)^{*}$ is an isomorphism of linear spaces.

Let us show that $\theta^{\prime}$ is an isomorphism of linear spaces. First let us check that $\theta^{\prime}$ is injective. Assume that $\psi \in \operatorname{Ker} \theta^{\prime}$. Since $V_{c}^{\infty}(X) \subset V_{c}^{-\infty}(X)$ then for any $\phi \in V_{c}^{\infty}(X)$ one has

$$
\int \phi \cdot \psi=0
$$

By the Selfduality Property (Theorem 6.1.1) $\psi \equiv 0$.

Let us show that $\theta^{\prime}$ is surjective. Let $\zeta \in V_{c}^{-\infty}(X)^{*}$. For any compact subset $K \subset X$ let us fix a compactly supported valuation $\gamma_{K} \in V_{c}^{\infty}(X)$ such that the restriction of $\gamma_{K}$ to a neighborhood of $K$ is equal to the Euler characteristic $\chi$. Consider the linear functional

$$
\zeta_{K}: V^{-\infty}(X) \rightarrow \mathbb{C}
$$

defined by $\zeta_{K}(\phi)=\zeta\left(\gamma_{K} \cdot \phi\right)$. It is easy to see that $\zeta_{K}$ is a continuous functional on $V^{-\infty}(X)$ equipped with the weak topology. Hence $\zeta_{K} \in V_{c}^{-\infty}(X)^{*}=V_{c}^{\infty}(X)$. It is also clear that if $K_{1} \subset K_{2}$ then the restriction of $\zeta_{K_{2}}$ to $K_{1}$ is equal to $\zeta_{K_{1}}$. Taking limit over all compact subsets of $X$ we get a smooth valuation on $X$ denoted by $\tilde{\zeta}$. Clearly the restriction of $\tilde{\zeta}$ to any compact subset $K \subset X$ is equal to $\zeta_{K}$. Then evidently $\zeta=\theta^{\prime}(\tilde{\zeta})$. Q.E.D. 


\subsection{The Euler-Verdier involution on generalized valuations.}

We are going to extend the Euler-Verdier involution from smooth valuations to generalized ones.

7.4.1 Theorem. (i) There exists unique continuous in the weak topology linear map

$$
\sigma: V^{-\infty}(X) \rightarrow V^{-\infty}(X)
$$

such that the restriction of it to $V^{\infty}(X)$ is the Euler-Verdier involution on smooth valuations.

(ii) $\sigma^{2}=I d$.

(iii) $\sigma$ commutes with the restrictions to open subsets of $X$, and thus induces an involution of the sheaf $\mathcal{V}_{X}^{-\infty}$ of generalized valuations (defined in Subsection [7.2).

(iv) $\sigma\left(W_{i}\left(V^{-\infty}(X)\right)\right)=W_{i}\left(V^{-\infty}(X)\right)$ for any $i=0,1, \ldots, n$.

(v) For any $\phi \in V^{\infty}(X), \xi \in V^{-\infty}(X)$ one has

$$
\sigma(\phi \cdot \xi)=\sigma(\phi) \cdot \sigma(\xi)
$$

Proof. Let us prove first part (i) The uniqueness is obvious since $V^{\infty}(X)$ is dense in $V^{-\infty}(X)$ in the weak topology. Let us probe the existence.

We have the Euler-Verdier involution on smooth valuations

$$
\sigma: V^{\infty}(X) \rightarrow V^{\infty}(X)
$$

Since this map commutes with restrictions to open subsets of $X$, it preserves support of a smooth valuation. Hence $\sigma: V_{c}^{\infty}(X) \rightarrow V_{c}^{\infty}(X)$ is a continuous operator (with respect to the topology of inductive limit on $\left.V_{c}^{\infty}(X)\right)$. Consider the dual operator

$$
\sigma^{*}: V^{-\infty}(X) \rightarrow V^{-\infty}(X)
$$

$\sigma^{*}$ is continuous in the weak topology. Let us show that the restriction of $(-1)^{n} \sigma^{*}$ to smooth valuations coincides with the Euler-Verdier involution on $V^{\infty}(X)$. This will finish the proof of part (i) since $(-1)^{n} \sigma^{*}$ is the operator we need (which will be denoted again by $\sigma$ ).

Let $\psi \in V^{\infty}(X) \subset V^{-\infty}(X)$. It is enough to show that for any $\phi \in V_{c}^{\infty}(X)$ one has

$$
<(-1)^{n} \sigma^{*} \psi, \phi>=<\sigma \psi, \phi>
$$

Using the automorphism property of the Euler-Verdier involution on smooth valuations (Theorem 4.1.4) we have

$$
\begin{array}{r}
<(-1)^{n} \sigma^{*} \psi, \phi>=(-1)^{n}<\psi, \sigma \phi>=(-1)^{n} \int \psi \cdot \sigma \phi= \\
\int \sigma(\psi \cdot \sigma \phi)=\int \sigma \psi \cdot \phi=<\sigma \psi, \phi>.
\end{array}
$$

Part (i) is proved. The remaining statements of the theorem follow from the continuity and the corresponding properties of the Euler-Verdier involution on smooth valuations. Q.E.D. 


\section{Valuations on real analytic manifolds.}

The goal of this section is to make a comparison of valuations with a more familiar space of constructible functions on a real analytic manifold. Let us fix a real analytic manifold $X$ of dimension $n$.

In Subsection 8.1 we construct a canonical imbedding of the space of constructible functions $\mathcal{F}(X)$ into the space of generalized valuations $V^{-\infty}(X)$ as a dense subspace. In Subsection 8.2 we show that the restriction of the canonical filtration on $V^{-\infty}(X)$ to $\mathcal{F}(X)$ is the filtration of $\mathcal{F}(X)$ by codimension of the support. In Subsection 8.3 it is proved that the restriction of the integration functional on the space of generalized valuations with compact support to the subspace $\mathcal{F}_{c}(X)$ of constructible functions with compact support is the integration with respect to the Euler characteristic. In Subsection 8.4 we show that the restriction of the Euler-Verdier involution on generalized valuation to $\mathcal{F}(X)$ coincides (up to a sign) with the Verdier duality operator on the latter.

\subsection{Imbedding of constructible functions to generalized valua- tions.}

In this subsection we will construct a canonical $\mathbb{C}$-linear map

$$
\Xi: \mathcal{F}(X) \rightarrow V^{-\infty}(X)
$$

and prove that it is injective and has a dense image in the weak topology, where $\mathcal{F}(X)$ is the space of constructible functions on $X$ defined in Subsection 1.2 (equality (1.2.1) ).

The construction of the map $\Xi$ is based on the notion of characteristic cycle attached to an arbitrary constructible function $f \in \mathcal{F}(X)$ denoted by $C C(f)$. This notion was discussed in Subsection 1.3.

Note in addition that the characteristic cycle satisfies

$$
C C(\alpha f+\beta g)=\alpha C C(f)+\beta C C(g)
$$

for any $\alpha, \beta \in \mathbb{C}$ (see [18, $\S 9.7)$.

Now let us describe the canonical map

$$
\Xi: \mathcal{F}(X) \rightarrow V^{-\infty}(X)=\left(V_{c}^{\infty}(X)\right)^{*}
$$

Let us denote by $C_{c}^{\infty}\left(T^{*} X, \Omega^{n} \otimes p^{*} o\right)$ the space of $C^{\infty}$-sections with compact support of the bundle $\Omega^{n} \otimes p^{*} o$ over $T^{*} X$. By Lemma 2.1.1 we have the canonical continuous epimorphism

$$
C_{c}^{\infty}\left(T^{*} X, \Omega^{n} \otimes p^{*} o\right) \rightarrow V_{c}^{\infty}(X)
$$

given by

$$
\omega \mapsto\left[P \mapsto \int_{C C(P)} \omega\right]
$$

for any $P \in \mathcal{P}(X)$. For any constructible function $f \in \mathcal{F}(X)$ let us define $\Xi(f)$ by

$$
<\Xi(f), \phi>=\int_{C C(f)} \omega
$$


where $\omega \in C_{c}^{\infty}\left(T^{*} X, \Omega^{n} \otimes p^{*} o\right)$ is an arbitrary lift of $\phi$. Once we show that $\Xi(f)$ is well defined, then automatically it is a continuous linear functional on $V_{c}^{\infty}(X)$.

Thus it remains to check that $\Xi$ is well defined. More explicitly, assume that $\omega \in$ $C_{c}^{\infty}\left(T^{*} X, \Omega^{n} \otimes p^{*} o\right)$ satisfies

$$
\int_{C C(P)} \omega=0
$$

for any $P \in \mathcal{P}(X)$. We have to check that

$$
\int_{C C(f)} \omega=0
$$

for any constructible function $f \in \mathcal{F}(X)$.

Let us fix such an $\omega$. By (8.1.1) it is enough to assume that $f$ is the indicator function of a subanalytic subset $Q$.

Let us observe first of all that (obviously) every point $x \in X$ has a compact subanalytic neighborhood (and also an open subanalytic neighborhood). Hence we can choose a compact subanalytic neighborhood $S$ of the support of $\omega$. It is enough to check that for any subanalytic subset $Q \subset S$ one has

$$
\int_{C C(Q)} \omega=0
$$

Any point $x \in X$ has a pair of subanalytic neighborhoods $U_{x} \subset V_{x}$ such that $U_{x}$ is compact, $V_{x}$ is open, and there exists a real analytic diffeomorphism $g_{x}: V_{x} \tilde{\rightarrow} \mathbb{R}^{n}$. Hence one can find a finite covering of $S$ by compact subanalytic subsets $\left\{U_{i}\right\}_{i}$, find open subanalytic subsets $\left\{V_{i}\right\}_{i}$ with $U_{i} \subset V_{i}$, and real analytic diffeomorphisms $f_{i}: V_{i} \stackrel{\sim}{\rightarrow} \mathbb{R}^{n}$.

By the linearity of the characteristic cycle (8.1.1), intersecting $Q$ with each $U_{i}$ we may assume that $Q$ is relatively compact subset of $V_{i_{0}}$ for some $i_{0}$. Thus it remains to prove the following statement.

8.1.1 Lemma. Let $\omega \in \tilde{C}^{\infty}\left(T^{*} \mathbb{R}^{n}, \Omega^{n} \otimes p^{*} o\right)$ satisfies

$$
\int_{C C(P)} \omega=0 \text { for any } P \in \mathcal{P}(X) .
$$

Then for any bounded subanalytic subset $Q \subset \mathbb{R}^{n}$ one has

$$
\int_{C C(Q)} \omega=0
$$

Proof. We will reduce the proof of the lemma to Theorem 1 of [7]. Let us fix an orientation on $\mathbb{R}^{n}$. Let $\psi$ denote the restriction of $\omega$ to the zero section $\underline{0}$ of $T^{*} X$. Thus $\psi \in C^{\infty}\left(\mathbb{R}^{n}, \Omega^{n}\right)$. Let

$$
q: T^{*} \mathbb{R}^{n} \backslash \underline{0} \rightarrow \mathbb{P}_{+}\left(T^{*} \mathbb{R}^{n}\right)
$$

be the canonical projection. Let $\tilde{\omega}:=q_{*} \omega$ be the integration of $\left.\omega\right|_{T^{*} \mathbb{R}^{n} \backslash \underline{0}}$ along the fibers of $q$. Let $a: \mathbb{P}_{+}\left(T^{*} \mathbb{R}^{n}\right) \rightarrow \mathbb{P}_{+}\left(T^{*} \mathbb{R}^{n}\right)$ be the canonical (antipodal) involution described in 
Subsection 1.3. Set $\eta:=a^{*} \tilde{\omega}$. It is easy to see that

$$
\begin{gathered}
\int_{C C(P)} \omega=\int_{N(P)} \eta+\int_{P} \psi \text { for any } P \in \mathcal{P}\left(\mathbb{R}^{n}\right) ; \\
\int_{C C(f)} \omega=\int_{N(f)} \eta+\int_{\mathbb{R}^{n}} f \cdot \psi \text { for any } f \in \mathcal{F}_{c}\left(\mathbb{R}^{n}\right) .
\end{gathered}
$$

Thus by assumption we get

$$
\int_{N(P)} \eta+\int_{P} \psi=0
$$

for any $P \in \mathcal{P}\left(\mathbb{R}^{n}\right)$.

It was shown in [7], Theorem 1 , that a pair $(\eta, \psi)$ with $\eta \in C^{\infty}\left(\mathbb{P}_{+}\left(T^{*} \mathbb{R}^{n}\right), \Omega^{n-1}\right), \psi \in$ $C^{\infty}\left(\mathbb{R}^{n}, \Omega^{n}\right)$ satisfies the equality (8.1.8) for any compact subanalytic subset $P$ if and only if it satisfies the following two conditions (where $\pi: \mathbb{P}_{+}\left(T^{*} \mathbb{R}^{n}\right) \rightarrow \mathbb{R}^{n}$ is the canonical projection):

$$
\begin{array}{r}
\int_{\pi^{-1}(x)} \eta=0 \text { for any } x \in \mathbb{R}^{n}, \\
D \eta+\pi^{*} \psi=0
\end{array}
$$

where $D: C^{\infty}\left(\mathbb{P}_{+}\left(T^{*} \mathbb{R}^{n}\right), \Omega^{n-1}\right) \rightarrow C^{\infty}\left(\mathbb{P}_{+}\left(T^{*} \mathbb{R}^{n}\right), \Omega^{n}\right)$ is an explicitly written differential operator of second order (introduced by Rumin in [22]).

However in the proof of the "if" part of Theorem 1 in [7] the authors used equality (8.1.8) not for the whole class of compact subanalytic sets, but for the subclass of compact subanalytic submanifolds with boundary. Hence if (8.1.8) is satisfied for all $P \in \mathcal{P}\left(\mathbb{R}^{n}\right)$ then the conditions (8.1.9), (8.1.10) are satisfied, and hence (8.1.8) is satisfied for an arbitrary compact subanalytic subset $P \subset \mathbb{R}^{n}$ (again by Theorem 1 of [7]).

In order to prove our lemma it is enough to show that (8.1.8) is satisfied for any bounded subanalytic subset $P$. Then we have

$$
\int_{C C\left(\mathbf{1}_{P}\right)} \omega=\int_{C C\left(\mathbf{1}_{\bar{P}}\right)} \omega-\int_{C C\left(\mathbf{1}_{\bar{P} \backslash P}\right)} \omega=-\int_{C C\left(\mathbf{1}_{\bar{P} \backslash P}\right)} \omega .
$$

Since $\operatorname{dim}(\bar{P} \backslash P)<\operatorname{dim} P$ by Proposition 1.2.5(ii) we can use the induction on the dimension of $P$. Lemma is proved. Q.E.D.

8.1.2 Remark. The differential operator $D$ was introduced by Rumin 22 for an arbitrary contact manifold, and it depends only on the contact structure. In our case for any smooth manifold $X$ the space $\mathbb{P}_{+}\left(T^{*} X\right)$ has a canonical contact structure, and the operator $D$ used in the proof of Lemma 8.1.1 corresponds to it.

\subsection{Comparison of filtrations.}

Let us define on $\mathcal{F}(X)$ a filtration by codimension of support:

$$
W_{i}(\mathcal{F}(X)):=\{f \in \mathcal{F}(X) \mid \operatorname{codim}(\operatorname{supp} f) \geq i\} .
$$

We have

$$
\mathcal{F}(X)=W_{0}(\mathcal{F}(X)) \supset W_{1}(\mathcal{F}(X)) \supset \cdots \supset W_{n}(\mathcal{F}(X)) \supset W_{n+1}(\mathcal{F}(X))=0 .
$$


8.2.1 Proposition. The canonical map

$$
\Xi: \mathcal{F}(X) \rightarrow V^{-\infty}(X)
$$

is injective. Moreover for any $i=0,1, \ldots, n$, and any $f \in W_{i}(\mathcal{F}(X)) \backslash W_{i+1}(\mathcal{F}(X))$ there exists $\phi \in W_{n-i}\left(V_{c}^{\infty}(X)\right)$ such that

$$
<\Xi(f), \phi>\neq 0
$$

Proof. Clearly it is enough to prove the second statement. Let us fix a constructible function $f \in W_{i}(\mathcal{F}(X)) \backslash W_{i+1}(\mathcal{F}(X))$. Thus supp $f$ is a subanalytic set and codim $(\operatorname{supp} f)=i$.

One can choose a regular point $x \in \operatorname{supp} f$, a neighborhood $U$, a real analytic diffeomorphism $g: U \stackrel{\sim}{\rightarrow} \mathbb{R}^{n}$ such that $\left.f\right|_{U} \circ g^{-1}=c \cdot \mathbb{1}_{\mathbb{R}^{n-k}}$ where $\mathbb{R}^{n-k} \subset \mathbb{R}^{n}$ is the coordinate subspace, and $c \neq 0$ is a constant. Thus we may assume that $X=\mathbb{R}^{n}, f=\mathbb{1}_{\mathbb{R}^{n-k}}$. Let us choose $\omega \in C_{c}^{\infty}\left(T^{*} \mathbb{R}^{n}, \Omega^{n} \otimes p^{*} o\right)$ as follows. Let $\left\{\left(q_{1}, \ldots, q_{n}\right)\right\}$ be coordinates on $\mathbb{R}^{n}$. Let $\left\{p_{1}, \ldots, p_{n}\right\}$ be dual coordinates on $\mathbb{R}^{n *}$. Let us fix a $C^{\infty}$-smooth non-negative compactly supported function $\tau: \mathbb{R}^{n *} \rightarrow \mathbb{R}_{\geq 0}$ such that $\tau(0)>0$. Let us take

$$
\omega:=\tau \cdot d x_{1} \wedge \cdots \wedge d x_{n-k} \wedge d y_{n-k+1} \wedge \cdots \wedge d y_{n}
$$

Then clearly $\int_{C C\left(\mathbb{R}^{n-k}\right)} \omega \neq 0$ and $\omega \in C_{c}^{\infty}\left(T^{*} \mathbb{R}^{n}, W_{n-i}\left(\Omega^{n} \otimes p^{*} o\right)\right)$. Q.E.D.

From now on we will identify $\mathcal{F}(X)$ with the subspace of $V^{-\infty}(X)$ via the imbedding $\Xi$.

8.2.2 Proposition. (i) $\mathcal{F}(X)$ is dense in $V^{-\infty}(X)$ in the weak topology.

(ii) For any $i=0,1, \ldots, n$

$$
\mathcal{F}(X) \cap W_{i}\left(V^{-\infty}(X)\right)=W_{i}(\mathcal{F}(X)) .
$$

Proof. (i) By the Hahn-Banach theorem it is enough to prove that for any $\phi \in$ $V_{c}^{\infty}(X) \backslash\{0\}$ there exists $f \in \mathcal{F}(X)$ such that $<f, \phi>\neq 0$. Let us fix $\phi \in V_{c}^{\infty}(X) \backslash\{0\}$. One may find an open subset $U \subset X$ and a real analytic diffeomorphism $g: U \stackrel{\sim}{\rightarrow} \mathbb{R}^{n}$ such that $\left.\phi\right|_{U} \not \equiv 0$. The smooth valuation $\left.g_{*} \phi\right|_{U} \in V^{\infty}\left(\mathbb{R}^{n}\right)$ does not vanish identically. By Proposition 2.4.10 from [5] there exists a convex compact set $K \in \mathcal{K}\left(\mathbb{R}^{n}\right) \cap \mathcal{P}\left(\mathbb{R}^{n}\right)$ such that $\left(g_{*} \phi\right)(K) \neq 0$. Since every compact set can be approximated in the Hausdorff metric by convex compact polytopes, we may assume that $K$ is a convex compact polytope, and hence a subanalytic set. Hence $g^{-1}(K)$ is a compact subanalytic subset of $X$. Take $f:=\mathbb{1}_{g^{-1}(K)}$. Then

$$
<f, \phi>\neq 0 \text {. }
$$

Part (i) is proved.

(ii) First let us show the inclusion

$$
\mathcal{F}(X) \cap W_{i}\left(V^{-\infty}(X)\right) \subset W_{i}(\mathcal{F}(X))
$$

Let $f \in \mathcal{F}(X)$ be such that $f \notin W_{i}(\mathcal{F}(X))$. Let us choose $l<i$ such that $f \in W_{l}(\mathcal{F}(X)) \backslash W_{l+1}(\mathcal{F}(X))$. By Proposition 8.2.1 there exists $\phi \in W_{n-l}\left(V_{c}^{\infty}(X)\right)$ such that $<f, \phi>\neq 0$. Hence $f \notin$ $W_{l+1}\left(V^{-\infty}(X)\right)$. Since $l+1 \leq i$ we have $f \notin W_{i}\left(V^{-\infty}(X)\right)$. This proves the inclusion (8.2.2). 
Let us prove the opposite inclusion

$$
W_{i}(\mathcal{F}(X)) \subset \mathcal{F}(X) \cap W_{i}\left(V^{-\infty}(X)\right) .
$$

By Proposition 7.3 .2 it is enough to show that for any $f \in W_{i}(\mathcal{F}(X)), \phi \in W_{n-i+1}\left(V_{c}^{\infty}(X)\right)$

$$
<f, \phi>=0 \text {. }
$$

By Lemma 2.1.1 and 8.1.1 there exists a compactly supported form $\omega \in C_{c}^{\infty}\left(T^{*} X, W_{n-i+1}\left(\Omega^{n} \otimes\right.\right.$ $\left.p^{*} o\right)$ ) such that for any $h \in \mathcal{F}_{c}(X)$

$$
<h, \phi>=\int_{C C(h)} \omega .
$$

Since the form $\omega$ is compactly supported the equality (8.2.4) holds for any $h \in \mathcal{F}(X)$.

Let us assume now that $f=\mathbb{1}_{Q}$ where $Q$ is a subanalytic subset with $\operatorname{codim} Q \geq i$. We may assume that $Q$ relatively compact. We have to show that $\int_{C C(Q)} \omega=0$. It is enough to show that the restriction of $\omega$ to $\operatorname{supp}(C C(Q))$ vanishes. By Lemma 1.3.2 one can find a finite covering $\bar{Q}=\cup_{\alpha} Q_{\alpha}$ such that $C C(Q) \subset \cup_{\alpha} T_{Q_{\alpha}}^{*} X$. But since $\operatorname{codim} Q_{\alpha} \geq i$ it is obvious that the restriction of $\omega$ to $T_{Q_{\alpha}}^{*} X$ vanishes. The proposition is proved. Q.E.D.

\subsection{The integration functional vs. the integration with respect to the Euler characteristic.}

On the space $\mathcal{F}_{c}(X)$ we have the linear functional $\mathcal{F}_{c}(X) \rightarrow \mathbb{C}$ of integration with respect to the Euler characteristic which is uniquely characterized by the property $\mathbb{1}_{Q} \mapsto \chi(Q)$ for any compact subanalytic subset $Q$ (see [18, §9.7). For a function $f \in \mathcal{F}_{c}(X)$ we will denote the integral of $f$ with respect to the Euler characteristic by $\int f d \chi$.

Thus we have the canonical imbedding

$$
\mathcal{F}_{c}(X) \hookrightarrow V_{c}^{-\infty}(X) .
$$

8.3.1 Proposition. The restriction of the integration functional $\int: V_{c}^{-\infty} \rightarrow \mathbb{C}$ to $\mathcal{F}_{c}(X)$ is equal to the integration with respect to the Euler characteristic.

Proof. Since the integration functional $\int: V_{c}^{-\infty}(X) \rightarrow \mathbb{C}$ to $\mathcal{F}_{c}(X)$ is continuous in the weak topology, Proposition 7.3 .10 implies that there exists unique $\xi \in V^{\infty}(X)$ such that for any $\psi \in V_{c}^{-\infty}(X)$

$$
\int \psi=<\psi, \xi>
$$

It is clear that if $\psi \in V_{c}^{\infty}(X)$ then

$$
\int \psi=<\psi, \chi>
$$

Since $V_{c}^{\infty}(X)$ is dense in $V_{c}^{-\infty}(X)$ by Proposition 7.3 .4 it follows that $\xi=\chi$.

Let us fix a Riemannian metric on $X$. By Theorems 1.5, 1.8 of [1] there exists a form $\omega \in \tilde{C}^{\infty}\left(T^{*} X, \Omega^{n} \otimes p^{*} o\right)$ (which is a little modification of the Chern-Gauss-Bonnet form [9]) 
such that for any compact subset $P \subset X$ which is either subanalytic or belongs to $\mathcal{P}(X)$ one has

$$
\chi(P)=\int_{C C(P)} \omega .
$$

Then by the construction of the imbedding $\mathcal{F}(X) \hookrightarrow V^{-\infty}(X)$ and by Proposition 1.2.7(ii) we have for any $f \in \mathcal{F}_{c}(X)$

$$
\int f=<f, \chi>=\int_{C C(f)} \omega .
$$

The proposition is proved. Q.E.D.

\subsection{The Euler-Verdier involution vs. the Verdier duality.}

The space of constructible functions $\mathcal{F}(X)$ has a canonical operator

$$
\mathbb{D}: \mathcal{F}(X) \rightarrow \mathcal{F}(X)
$$

called the Verdier duality (see [18], §9.7). It satisfies $\mathbb{D}^{2}=I d$, and for any function $f \in \mathcal{F}(X)$

$$
C C(\mathbb{D} f)=a^{*} C C(f)
$$

where $a: T^{*} X \rightarrow T^{*} X$ is the antipodal involution (Proposition 9.4.4 of [18]). The main result of this subsection is the following proposition.

8.4.1 Proposition. The restriction of the Euler-Verdier involution $\sigma: V^{-\infty}(X) \rightarrow V^{-\infty}(X)$ to $\mathcal{F}(X)$ is equal to $(-1)^{n} \mathbb{D}$.

Proof. Let $f \in \mathcal{F}(X)$. We have to show that for any $\phi \in V_{c}^{\infty}(X)$ one has

$$
<\sigma f, \phi>=(-1)^{n}<\mathbb{D} f, \phi>
$$

By Lemma 2.1.1 there exists $\omega \in C_{c}^{\infty}\left(T^{*} X, \Omega^{n} \otimes p^{*} o\right)$ such that for any $h \in \mathcal{F}(X)$ one has

$$
<h, \phi>=\int_{C C(h)} \omega .
$$

Then by the definition of $\sigma$ on $V^{-\infty}(X)$ we get

$$
\begin{array}{r}
<\sigma f, \phi>=(-1)^{n}<f, \sigma \phi>=\int_{C C(f)} a^{*} \omega= \\
(-1)^{n} \int_{C C(\mathbb{D} f)} \omega=<(-1)^{n} \mathbb{D} f, \phi>.
\end{array}
$$

The proposition is proved. Q.E.D. 


\section{References}

[1] Alesker, Semyon; Integrals of smooth and analytic functions over Minkowski's sums of convex sets. MSRI "Convex Geometric Analysis" 34 (1998), 1-15.

[2] Alesker, Semyon; Description of translation invariant valuations on convex sets with solution of P. McMullen's conjecture. Geom. Funct. Anal. 11 (2001), no. 2, 244-272.

[3] Alesker, Semyon; The multiplicative structure on polynomial continuous valuations. Geom. Funct. Anal. 14 (2004), no. 1, 1-26, also: math.MG/0301148.

[4] Alesker, Semyon; Theory of valuations on manifolds, I. Linear spaces. math.MG/0503397.

[5] Alesker, Semyon; Theory of valuations on manifolds, II. math.MG/0503399.

[6] Alesker, Semyon; Fu, Joseph H.G.; Theory of valuations on manifolds, III. math.MG/0509512

[7] Bernig, Andreas; Bröcker, Ludwig; Valuations on manifolds and Rumin cohomology. Preprint 2005.

[8] Bierstone, Edward; Milman, Pierre D.; Semianalytic and subanalytic sets. Inst. Hautes Études Sci. Publ. Math. No. 67 (1988), 5-42.

[9] Chern, Shiing-shen; On the curvatura integra in a Riemannian manifold. Ann. of Math. (2) 46, (1945). 674-684.

[10] Fu, Joseph H. G.; Kinematic formulas in integral geometry. Indiana Univ. Math. J. 39 (1990), no. 4, 1115-1154.

[11] Fu, Joseph H. G.; Curvature measures of subanalytic sets. Amer. J. Math. 116 (1994), no. $4,819-880$.

[12] Guillemin, Victor; Sternberg, Shlomo; Geometric asymptotics. Mathematical Surveys, No. 14. American Mathematical Society, Providence, R.I., 1977.

[13] Godement, Roger; Topologie algébrique et théorie des faisceaux. (French) Troisième édition revue et corrigée. Publications de l'Institut de Mathématique de l'Université de Strasbourg, XIII. Actualités Scientifiques et Industrielles, No. 1252. Hermann, Paris, 1973.

[14] Hardt, Robert M.; Stratification of real analytic mappings and images. Invent. Math. 28 (1975), 193-208.

[15] Hardt, Robert M.; Triangulation of subanalytic sets and proper light subanalytic maps. Invent. Math. 38 (1976/77), no. 3, 207-217.

[16] Hironaka, Heisuke; Subanalytic sets. Number theory, algebraic geometry and commutative algebra, in honor of Yasuo Akizuki, pp. 453-493. Kinokuniya, Tokyo, 1973. 
[17] Hironaka, Heisuke; Introduction to real-analytic sets and real-analytic maps. Quaderni dei Gruppi di Ricerca Matematica del Consiglio Nazionale delle Ricerche. Istituto Matematico "L. Tonelli" dell'Università di Pisa, Pisa, 1973.

[18] Kashiwara, Masaki; Schapira, Pierre; Sheaves on manifolds. With a chapter in French by Christian Houzel. Grundlehren der Mathematischen Wissenschaften [Fundamental Principles of Mathematical Sciences], 292. Springer-Verlag, Berlin, 1990.

[19] McMullen, Peter; Valuations and Euler-type relations on certain classes of convex polytopes. Proc. London Math. Soc. (3) 35 (1977), no. 1, 113-135.

[20] McMullen, Peter; Valuations and dissections. Handbook of convex geometry, Vol. A, B, 933-988, North-Holland, Amsterdam, 1993.

[21] McMullen, Peter; Schneider, Rolf; Valuations on convex bodies. Convexity and its applications, 170-247, Birkhäuser, Basel, 1983.

[22] Rumin, Michel; Formes différentielles sur les variétés de contact. J. Differential Geom. 39 (1994), no. 2, 281-330.

[23] Schneider, Rolf; Convex bodies: the Brunn-Minkowski theory. Encyclopedia of Mathematics and its Applications, 44. Cambridge University Press, Cambridge, 1993.

[24] Tamm, Martin; Subanalytic sets in the calculus of variation. Acta Math. 146 (1981), no. 3-4, 167-199.

[25] Wintgen, Peter; Normal cycle and integral curvature for polyhedra in riemannian manifolds. Differential Geometry (G. Soos and J. Szenthe, eds.), North-Holland, Amsterdam, 1982.

[26] Zähle, Martina; Curvatures and currents for unions of sets with positive reach. Geom. Dedicata 23 (1987), no. 2, 155-171. 